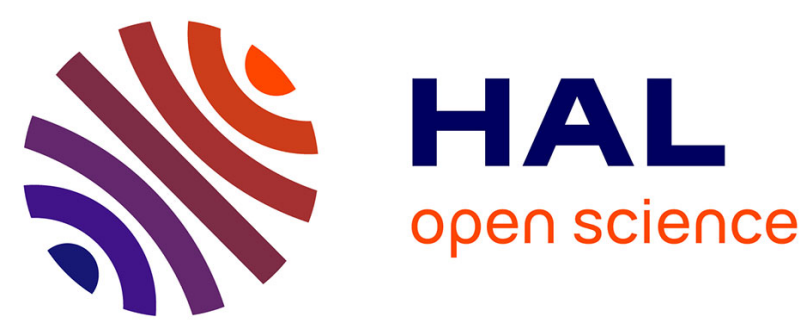

\title{
Thermodynamic modeling of the condensable fraction of a gaseous effluent from lignocellulosic biomass torrefaction
}

\author{
Mylène Detcheberry, Philippe Destrac, Silvère Massebeuf, Olivier Baudouin, \\ Vincent Gerbaud, Jean-Stéphane Condoret, Xuan Mi Meyer
}

\section{To cite this version:}

Mylène Detcheberry, Philippe Destrac, Silvère Massebeuf, Olivier Baudouin, Vincent Gerbaud, et al.. Thermodynamic modeling of the condensable fraction of a gaseous effluent from lignocellulosic biomass torrefaction. Fluid Phase Equilibria, 2016, 409, pp.242-255. 10.1016/j.fluid.2015.09.025 . hal-01338876

\section{HAL Id: hal-01338876 https://hal.science/hal-01338876}

Submitted on 12 Jul 2016

HAL is a multi-disciplinary open access archive for the deposit and dissemination of scientific research documents, whether they are published or not. The documents may come from teaching and research institutions in France or abroad, or from public or private research centers.
L'archive ouverte pluridisciplinaire $\mathbf{H A L}$, est destinée au dépôt et à la diffusion de documents scientifiques de niveau recherche, publiés ou non, émanant des établissements d'enseignement et de recherche français ou étrangers, des laboratoires publics ou privés. 


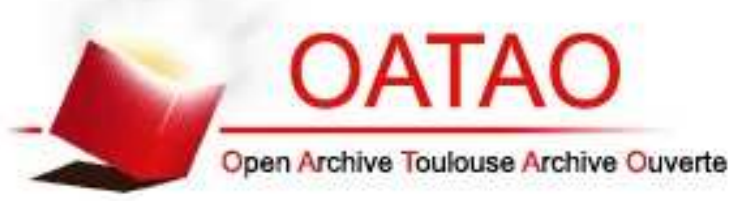

\section{Open Archive TOULOUSE Archive Ouverte (OATAO)}

OATAO is an open access repository that collects the work of Toulouse researchers and makes it freely available over the web where possible.

This is an author-deposited version published in : http://oatao.univ-toulouse.fr/ Eprints ID : 15861

To link to this article : DOI : 10.1016/j.fluid.2015.09.025

URL : http://dx.doi.org/10.1016/j.fluid.2015.09.025

To cite this version : Detcheberry, Mylène and Destrac, Philippe and Massebeuf, Silvère and Baudouin, Olivier and Gerbaud, Vincent and Condoret, Jean-Stéphane and Meyer, Xuân-Mi Thermodynamic modeling of the condensable fraction of a gaseous effluent from lignocellulosic biomass torrefaction. (2016) Fluid Phase Equilibria, vol. 409. pp. 242-255. ISSN 0378-3812

Any correspondence concerning this service should be sent to the repository administrator: staff-oatao@ listes-diff.inp-toulouse.fr 


\title{
Thermodynamic modeling of the condensable fraction of a gaseous effluent from lignocellulosic biomass torrefaction
}

\author{
M. Detcheberry ${ }^{\text {a, b }}$, P. Destrac ${ }^{\text {a, b }}$, S. Massebeuf ${ }^{\text {c }}$, O. Baudouin ${ }^{\text {c }}$, V. Gerbaud ${ }^{\text {a, b }}$, \\ J.-S. Condoret ${ }^{\mathrm{a}, \mathrm{b}}$, X.-M. Meyer ${ }^{\mathrm{a}, \mathrm{b}}$, * \\ a Université de Toulouse, INPT, UPS, Laboratoire de Génie Chimique, 4, Allée Emile Monso, F-31030 Toulouse, France \\ ${ }^{b}$ CNRS, Laboratoire de Génie Chimique, F-31030 Toulouse, France \\ ${ }^{\mathrm{c}}$ ProSim SA, Immeuble Stratège A, 51 rue Ampère, F-31670 Labège, France
}

Keywords:

Reactive phase equilibria

Modeling

Torrefaction

Volatile matter

UNIQUAC

\begin{abstract}
A B S T R A C T
The condensable fraction of the gaseous effluent from the torrefaction process of wood is a complex mixture of more than one hundred oxygenated species (alcohols, acids, aldehydes, ketones, furans, phenolic, gaïacols and sugars) diluted in water where some of them are likely to react. This effluent is currently burnt to provide energy but it could be valorized as bio-sourced chemicals. To recover target products like acetic acid, glycolaldehyde, furfural and eugenol a first step of thermodynamic modeling of this complex mixture is required to be able to propose different strategies of separation-purification. This was done here by coupling the UNIQUAC model with chemical equilibria involved in the reactive mixture. Binary interaction parameters were identified using vapor-liquid equilibria data from the literature. The predicted results are in good agreement with the experimental data of systems containing water, methanol, formaldehyde, acetic acid, formic acid, propionic acid, furfural and furfuryl alcohol, main components of the considered mixture and their associated reaction products.
\end{abstract}

\section{Introduction}

Sustainable resources and processes are nowadays increasingly studied to propose alternatives to the use of fossil raw materials. Lignocellulosic biomass, as wood for example, is a renewable resource but its moisture content is high and it is not an easily grindable material [1]. Furthermore, its energy density is lower than coal. These issues could be overcome thanks to the torrefaction process.

Torrefaction is a thermal process carried out at temperatures below $300{ }^{\circ} \mathrm{C}$, under inert atmosphere, at atmospheric pressure, and with residence times for the solid biomass ranging from few minutes to several hours [2,3]. Torrefied wood is a solid product constituted by more than $70 \%$ of the initial mass with properties close to those of coal. The $30 \%$ remaining part is a gaseous effluent [2,3], composed of about one third of non condensable gases carbon monoxide and carbon dioxide - and two thirds of condensable species.

\footnotetext{
* Corresponding author. Université de Toulouse, INPT, UPS, Laboratoire de Génie Chimique, 4, Allée Emile Monso, F-31030 Toulouse, France.

E-mail address: xuan.meyer@ensiacet.fr (X.-M. Meyer).
}

Currently, torrefied wood is the main product of interest and is usually transformed into energetic gases by the gasification process [4-6] or directly used as coal for combustion [7,8]. Conversely, gaseous by-products are considered at present time as a waste [9] and in the best case are burned to provide energy to the process [6]. Yet, the recovery and valorization of the condensable fraction as bio-sourced chemicals is worth considering.

An experimental study of the torrefaction of four various biomass types showed that there were significant differences in gaseous product composition depending on the nature of the biomass [10]. Condensable species composition exhibit more than one hundred oxygenated components (partially identified and quantified) and significantly differs depending on the biomass type.

Any preliminary study to assess new routes, as for instance non energetic valorization of such gaseous effluent, requires knowledge of thermodynamics of these complex mixtures. Indeed, some thermodynamic models already exist for part of this mixture. In the general biorefinery field, some experimental and modeling studies of vapor-liquid equilibria have been published [11,12]. More specifically, thermodynamics of formaldehyde (one of the major components of this gaseous effluent), and its mixtures with water, were developed using an approach coupling physical and chemical 
equilibria $[13,14]$.

This work is indeed an extension of our previously published model [13] with the aim at representing now the vapor-liquid thermodynamic behavior of the whole torrefaction condensable fraction using a combined physical and chemical model. In this paper, a strategy for modeling the vapor-liquid equilibria for a mixture of 22 representative components is proposed, including possible chemical reactions.

The paper is organized as follows. In Section 1 the characteristics of condensates from lignocellulosic biomass torrefaction are briefly introduced. In Section 2, the strategy to develop the thermodynamic model is exposed and the choice of UNIQUAC to calculate activity coefficients is justified. Section 3 presents the method to estimate the unknown UNIQUAC binary interaction parameters. In Section 4 the results are reported and discussed. Indeed, such a thermodynamic modeling is the pre-requisite to propose and assess (on energetic and economic criteria) different separation schemes to produce bio-sourced chemicals from the gaseous effluent of the torrefaction process. These future studies (not in the scope of this work), based on this modeling, will be able to provide the quantitative data to decide the viability of such a valorization strategy.

\section{Characterization of condensates from lignocellulosic biomass torrefaction}

Few descriptions of the volatile matter after torrefaction are available in the literature. Table 1 gives a short inventory of the species identified in torrefaction effluents. Non condensable gases are mainly carbon monoxide and carbon dioxide. A focus on the condensable part of the volatile matter shows that condensates are a multicomponent mixture, chemically and thermally unstable, containing oxygenated species diluted in water. The oxygenated species belong to different chemical classes: water, alcohols, acids, aldehydes, ketones, furans, phenolics, gaïacols.
The main component is water accounting for $60 \% \mathrm{~mol}$ to $80 \% \mathrm{~mol}$. Minor components are diluted in water which makes their separation a hard task. Moreover, minor components are present in proportions varying with the processed biomass [2].

As it is impossible to consider all the components present in condensates for modeling, a representative mixture was established for condensates. The analysis of the experimental data collected in the frame of INVERTO project enabled us to select an acceptable number of 22 components including: water $(W)$, methanol $(M E)$, formaldehyde $(F A)$, methylene glycol $(M G)$, hemiformal $(H F), 6$ poly(oxymethylene) glycols from a degree 2 to a degree $7\left(M G_{2}-M G_{7}\right), 6$ poly(oxymethylene) hemiformals from a degree 2 to a degree $7\left(H F_{2}-H F_{7}\right)$, acetic acid $\left(A_{1}\right)$, formic acid $\left(A_{2}\right)$, propionic acid $\left(A_{3}\right)$, furfural $(F u)$ and furfuryl alcohol $(F u A l)$. All these compounds are present in significant amounts (a few $\mathrm{g} / \mathrm{L}$ in the condensed aqueous phase).

A previous study was dedicated to the modeling of aqueous solutions of formaldehyde and methanol [13] and the same approach is used here to be extended to the modeling of the representative mixture of the torrefaction condensates.

\section{Thermodynamic model}

The complexity of the condensate mixture makes its purification a difficult task and this complexity has to be handled first by a suitable thermodynamic description. An important point to emphasize is the presence of reactive components in the mixture: carboxylic acids associate in the vapor phase and formaldehyde polymerizes with water and methanol to produce hemiformal, methylene glycol, poly(oxymethylene) hemiformals and poly(oxymethylene) glycols. So, vapor-liquid equilibria must be coupled with those chemical equilibria for a suitable description of condensates thermodynamic behavior.

Table 1

Inventory of species identified in torrefaction effluents listed in the literature.

\begin{tabular}{|c|c|c|c|c|c|c|c|c|c|}
\hline Chemical class & CAS number & Component & {$[1]$} & [15] & {$[16]$} & {$[17]$} & {$[2]$} & {$[10]$} & Our mixture \\
\hline Alcohol & $67-56-1$ & Methanol & $\checkmark$ & & & $\checkmark$ & $\checkmark$ & & $\checkmark$ \\
\hline \multirow[t]{4}{*}{ Aldehydes and Ketones } & $116-09-6$ & Hydroxyacetone (acetol) & $\checkmark$ & $\checkmark$ & & $\checkmark$ & & $\checkmark$ & $\checkmark$ \\
\hline & $75-07-0$ & Acetaldehyde & & & & & & $\checkmark$ & \\
\hline & $141-46-8$ & Hydroxyacetaldehyde (glycolaldehyde) & & $\checkmark$ & & & & $\checkmark$ & $\checkmark$ \\
\hline & $50-00-0$ & Formaldehyde & & $\checkmark$ & & & $\checkmark$ & $\checkmark$ & $\checkmark$ \\
\hline \multirow[t]{4}{*}{ Acids } & $64-19-7$ & Acetic acid & $\checkmark$ & $\checkmark$ & & $\checkmark$ & $\checkmark$ & $\checkmark$ & $\checkmark$ \\
\hline & $64-18-6$ & Formic acid & $\checkmark$ & $\checkmark$ & & $\checkmark$ & $\checkmark$ & $\checkmark$ & $\checkmark$ \\
\hline & $79-09-4$ & Propionic acid & & $\checkmark$ & & & & $\checkmark$ & $\checkmark$ \\
\hline & $50-21-5$ & Lactic acid & $\checkmark$ & & & $\checkmark$ & & & \\
\hline \multirow[t]{2}{*}{ Furans } & $98-01-1$ & Furfural & $\checkmark$ & & & $\checkmark$ & $\checkmark$ & $\checkmark$ & $\checkmark$ \\
\hline & $98-00-0$ & 2-furanmethanol & & & & & & $\checkmark$ & $\checkmark$ \\
\hline \multirow{14}{*}{ Phenolics and Gaïacols } & $108-95-2$ & Phenol & $\checkmark$ & & $\checkmark$ & & & & \\
\hline & $90-05-1$ & 2-methoxyphenol (gaïacol) & & & $\checkmark$ & & & & \\
\hline & $106-44-5$ & 4-methylphenol (p-cresol) & & & $\checkmark$ & & & & \\
\hline & $93-51-6$ & 2-methoxy-4-methylphenol (4-methylgaïcol) & & & $\checkmark$ & & & & \\
\hline & 2785-89-9 & 4-ethyl-2-methoxyphenol (4-ethylgaïacol) & & & $\checkmark$ & & & & \\
\hline & $91-10-1$ & 2.6-dimethoxyphenol (syringol) & & & $\checkmark$ & & & & \\
\hline & $97-53-0$ & 2-methoxy-4-prop-2-enylphenol (eugenol) & & & $\checkmark$ & & & & \\
\hline & $121-33-5$ & 4-hydroxy-3-methoxybenzaldehyde (vanillin) & & & $\checkmark$ & & & & \\
\hline & & 2-methoxy-4-(1E)-prop-1-en-1-ylphenol & & & $\checkmark$ & & & & \\
\hline & $121-34-6$ & 4-hydroxy-3-methoxybenzoic acid (vanillic acid) & & & $\checkmark$ & & & & \\
\hline & $6443-69-2$ & 1.2.3-trimethoxy-5-methylbenzene & & & $\checkmark$ & & & & \\
\hline & & 2.6-dimethoxy-4-prop-2-enylphenol & & & $\checkmark$ & & & & \\
\hline & & 1.4-hydroxy-3.5-dimethoxyphenylethanone & & & $\checkmark$ & & & & \\
\hline & $2478-38-8$ & 7.9-dihydroxy-3-methoxy-1-methyl-6H-dibenzo(b.d)pyran-6-one & & & $\checkmark$ & & & & \\
\hline Water & $7732-18-5$ & Water & $\checkmark$ & $\checkmark$ & & $\checkmark$ & $\checkmark$ & $\checkmark$ & $\checkmark$ \\
\hline \multirow[t]{2}{*}{ Incondensables } & $124-38-9$ & Carbon dioxide & $\checkmark$ & $\checkmark$ & & $\checkmark$ & $\checkmark$ & $\checkmark$ & \\
\hline & $630-08-0$ & Carbon monoxide & $\checkmark$ & $\checkmark$ & & $\checkmark$ & $\checkmark$ & $\checkmark$ & \\
\hline
\end{tabular}


3.1. Description of the thermodynamic behavior of the reactive mixture

As mentioned above, when modeling thermodynamics of such systems, the main difficulty is to account for the coupling of chemical and physical equilibria of these reactive molecules. A review of thermodynamics for reactive mixtures has been given by Maurer [14] where it is suggested to uncouple the physical and the chemical phenomena in the model so as to differenciate the effects of weak intermolecular interactions of the physical equilibria from the strong intermolecular interactions involved in the chemical reactions. This modeling approach has also the advantage of avoiding spreading the uncertainty on the chemical equilibrium constant into the physical equilibrium parameters.

Fig. 1 illustrates the outline of this model. Note that in our approach the system is considered at chemical and physical equilibrium and therefore no chemical or physical kinetic data are considered.

Thus, the reactive vapor-liquid equilibrium model includes:

- physical phase equilibria described using a $\gamma-\varphi$ approach to account for this multicomponent system with a large range of molar masses and volatilities. The physical interactions between all species are taken into account through activity coefficients calculation in the liquid phase and through an equation of state for the gas phase.

- 2 chemical reaction equilibria for the formation of methylene glycol and hemiformal:

- formation of methylene glycol: $F A+W \rightleftharpoons M G$

- formation of hemiformal: $F A+M E \rightleftharpoons H F$

- 12 chemical reaction equilibria between the poly(oxymethylene) glycols, poly(oxymethylene) hemiformals:

- formation of poly(oxymethylene) glycols: $M G_{n-1}+M G \rightleftharpoons M G_{n}+W$
- formation of poly(oxymethylene) hemiformals: $H F_{n-1}+H F \rightleftharpoons H F_{n}+M E$

- 3 direct dimerization and 3 crossed dimerization chemical equilibria of acetic acid, formic acid and propionic acid are assumed to occur in the vapor phase:

- direct dimerization of a carboxylic acid $A_{i}: 2 A_{i} \rightleftharpoons A_{i 2}$

- crossed dimerization of $A_{i}$ and $A_{j}: A_{i}+A_{j} \rightleftharpoons A_{i} A_{j}$

where $A_{i}$ and $A_{j}$ correspond to one of the following carboxylic acid: acetic acid, formic acid, propionic acid. A total of 20 reactions are accounted for in the description of the behavior of the reactive mixture.

Note that once the vapor-liquid equilibrium equations and the chemical reaction equilibrium equations in one phase are satisfied, the chemical-reaction equilibrium equations in the other phase are automatically satisfied.

\subsection{Vapor-liquid equilibrium model}

As the model will not be used under pressure, the gas phase was considered as a perfect gas where gas phase associations of carboxylic acids; and methylene glycol and hemiformal formations are included.

To calculate the activity coefficients of the liquid phase, three models based on the local composition were considered: UNIversal Functional Activity Coefficient Original (UNIFAC Original). Non Random Two Liquids (NRTL) and UNIversal QUAsi Chemical (UNIQUAC). Table 2 synthetises a comparison of these thermodynamic models. The advantage of the UNIFAC Original model lies in its predictive capability and is interesting when experimental data are lacking. Meanwhile, its range of temperature applicability is relatively poor [18]. Pressure and temperature ranges of UNIQUAC applicability are greater than UNIFAC Original. Semi-empirical models like NRTL or UNIQUAC are more accurate for the binaries

$$
\begin{array}{ll}
A_{1}+A_{2} \Leftrightarrow A_{1} A_{2} & 2 A_{1} \Leftrightarrow A_{12} \\
A_{1}+A_{3} \Leftrightarrow A_{1} A_{3} & 2 A_{2} \Leftrightarrow A_{22} \\
A_{2}+A_{3} \Leftrightarrow A_{2} A_{3} & 2 A_{3} \Leftrightarrow A_{32} \\
F A+W \Leftrightarrow M G & \\
F A+M E \Leftrightarrow H F &
\end{array}
$$

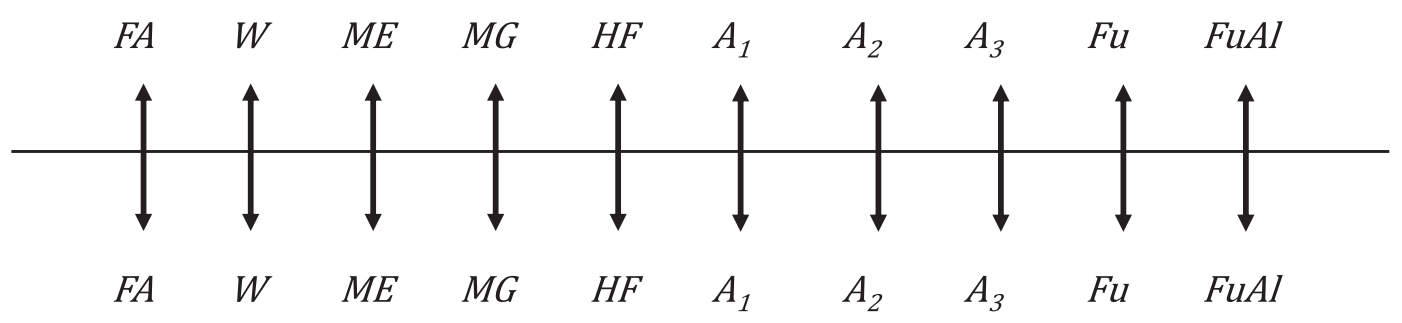

$$
\begin{aligned}
& M G_{n-1}+M G \Leftrightarrow M G_{n}+W \\
& H F_{n-1}+H F \Leftrightarrow H F_{n}+M E
\end{aligned}
$$


Table 2

Comparison of thermodynamics models based on the local composition concept.

\begin{tabular}{|c|c|c|}
\hline Thermodynamic model & Advantages & Drawbacks \\
\hline UNIFAC Original & $\begin{array}{l}\text { No experimental data required (predictive model) } \\
\text { Multicomponent vapor-liquid equilibria }\end{array}$ & $\begin{array}{l}\text { Not recommended for process design } \\
\text { Not able to differenciate isomers } \\
\text { Poor range of temperature applicability }\end{array}$ \\
\hline NRTL & $\begin{array}{l}\text { Multicomponent vapor-liquid and liquid-liquid equilibria } \\
\text { Widely used for flowsheeting and process design } \\
\text { Experimental data taken into account in the model }\end{array}$ & $\begin{array}{l}\text { Large number of binary interaction parameters to identify } \\
\text { Molecule shape and size difference effects not taken into account }\end{array}$ \\
\hline UNIQUAC & $\begin{array}{l}\text { Multicomponent vapor-liquid and liquid-liquid equilibria } \\
\text { Widely used for flowsheeting and process design } \\
\text { Experimental data taken into account in the model } \\
\text { Molecule shape and size difference effects taken into account directly in } \\
\text { the model }\end{array}$ & Large number of binary interaction parameters to identify \\
\hline
\end{tabular}

for which experimental data are available. Compared to NRTL, UNIQUAC takes into account the molecule shape and size difference effects and is then more suitable for the studied asymetric mixture containing both small molecules (methanol, formaldehyde, formic acid ...) and larger ones (furfural, poly(oxymethylene) glycols ...).

Therefore, the UNIQUAC model was selected to describe the non-ideality of the liquid phase. UNIQUAC equations [19] are given by:

$\ln \gamma_{i}=\ln \gamma_{i}^{C}+\ln \gamma_{i}^{R}$

$\ln \gamma_{i}^{C}=\ln \frac{\phi_{i}}{x_{i}}+\frac{Z}{2} \ln \frac{\theta_{i}}{\phi_{i}}+l_{i}-\frac{\phi_{i}}{x_{i}} \sum_{j=1}^{n_{\complement}} x_{j} l_{j}$

$\ln \gamma_{i}^{R}=q_{i}\left(1-\ln \sum_{j=1}^{n_{C}} \theta_{j} \tau_{j i}-\sum_{j=1}^{n_{c}} \frac{\theta_{i} \tau_{i j}}{\sum_{k=1}^{n_{c}} \theta_{k} \tau_{k j}}\right)$

$\phi_{i}=\frac{x_{i} r_{i}}{\sum_{i=1}^{n_{C}} x_{i} r_{i}}$ and $\theta_{i}=\frac{x_{i} q_{i}}{\sum_{i=1}^{n_{C}} x_{i} q_{i}}$

$l_{i}=\frac{Z}{2}\left(r_{i}-q_{i}\right)-\left(r_{i}-1\right)$

$\tau_{i j}=\exp \left(-\frac{A_{i j}}{R T}\right)$ and $\tau_{j i}=\exp \left(-\frac{A_{j i}}{R T}\right)$

$A_{i j}=A_{i j}^{0}+A_{i j}^{T}\left(T-T_{r e f}\right)$ and $A_{j i}=A_{j i}^{0}+A_{j i}^{T}\left(T-T_{r e f}\right)$

The UNIQUAC binary interaction parameters (see equation (7)) were identified for the different binaries of the condensate representative system using either experimental vapor-liquid data from literature when available or numerical data generated by UNIFAC Original $[20,21]$. The determination of UNIQUAC binary interaction parameters is detailed in the following part.

\section{Determination of UNIQUAC binary interaction parameters}

UNIQUAC binary interaction parameters must be estimated for systems including water, methanol, formaldehyde, methylene glycol, hemiformal, poly(oxymethylene) glycols $\left(M G-M G_{7}\right)$, poly(oxymethylene) hemiformals ( $\mathrm{HF}-\mathrm{HF}_{7}$ ), acetic acid, formic acid, propionic acid, furfural, furfuryl alcohol. The binary interaction parameters of the formaldehyde - water - methanol reactive system were formerly identified [13]. Some binaries (like water methanol, water - acetic acid ...) have been widely studied in the literature. Nevertheless, as the reported binary interaction parameters may have been estimated with other values of the pure component properties than those used in this study, they were identified again in this work using experimental data recommended by the DECHEMA.

Different cases were considered for the binary interaction parameters estimation (see Table 3 ):

1. case 1 : non-reactive binary systems

2. case 2: binary systems involving formaldehyde species and other components except carboxylic acids

3. case 3: binary systems involving carboxylic acids

Component abbreviations are defined in the nomenclature. The reactive vapor-liquid equilibrium of the water-methanolformaldehyde system was already modeled [13] so the reference of the publication is given for theses binaries.

For case 1 and case 2 , the same physical phase equilibrium equations were used, given by:

$y_{i} P=x_{i} \gamma_{i}(T, x) P_{i}^{S}(T)$

The coefficients $a_{i}$ of the equations to calculate the vapor pressure of pure component $i$ with respect to temperature were taken from the DIPPR Database [22] available through the Simulis Thermodynamics package $\ln P_{i}^{S}(T)=a_{1, S}+\frac{a_{2, S}}{T}+a_{3, S} \ln T+a_{4, S} T^{a_{5, S}}$.

When available in the literature experimental vapor-liquid data were used for the identification of the UNIQUAC binary interaction parameters. When no data were available, vapor-liquid numerical data at constant vapor ratio and temperature (or pressure) were generated using the UNIFAC Original model $[21,20]$. As mentioned above, it was chosen to uncouple the physical and chemical phenomena for systems involving formaldehyde species to avoid to spread the uncertainty of the chemical equilibrium constants into the parameters of the physical equilibrium. Because of the presence of the chemical reactions, no uncoupled vapor-liquid experimental data were available in the literature. So, vapor-liquid data were generated using the UNIFAC Original model for case 2. Note that binary interaction parameters of systems labeled 2 in Table 3 have to be ascertained as soon as experimental data become available.

Finally, for case 3 (binaries involving carboxylic acids), conventional physical phase equilibrium equations, with an association term to account for chemical equilibria, were used for the identification. Nevertheless, when dealing with the dimerization equilibria of carboxylic acids, it was found more convenient not to uncouple physical and chemical equilibria. Indeed, for these specific compounds, the uncoupled approach has been developed for long [23] and was already implemented in the Prosim Plus software. In this case, it is proposed to use a correction term in equation 
Table 3

Matrix of the modeling assumptions for each binary of the mixture.

\begin{tabular}{|c|c|c|c|c|c|c|c|c|c|c|c|c|}
\hline & $A_{1}$ & $A_{2}$ & $A_{3}$ & W & $M E$ & $F A$ & $M G$ & $M G_{2}-M G_{7}$ & $H F$ & $H F_{2}-H F_{7}$ & $F u$ & FuAl \\
\hline$A_{1}$ & - & - & - & - & - & - & - & - & - & - & - & - \\
\hline$A_{2}$ & 3 & - & - & - & - & - & - & - & - & - & - & - \\
\hline$A_{3}$ & 3 & 3 & - & - & - & - & - & - & - & - & - & - \\
\hline W & 3 & 3 & 3 & - & - & - & - & - & - & - & - & - \\
\hline$M E$ & 3 & 3 & 3 & 1 & - & - & - & - & - & - & - & - \\
\hline$F A$ & 3 & 3 & 3 & [13] & [13] & - & - & - & - & - & - & - \\
\hline$M G$ & 3 & 3 & 3 & [13] & [13] & [13] & - & - & - & - & - & - \\
\hline$M G_{2}-M G_{7}$ & 3 & 3 & 3 & [13] & [13] & [13] & [13] & - & - & - & - & - \\
\hline$H F$ & 3 & 3 & 3 & [13] & [13] & [13] & [13] & [13] & - & - & - & - \\
\hline$H F_{2}-H F_{7}$ & 3 & 3 & 3 & [13] & [13] & [13] & [13] & [13] & [13] & - & - & - \\
\hline$F u$ & 3 & 3 & 3 & 1 & 1 & 2 & 2 & 2 & 2 & 2 & - & - \\
\hline FuAl & 3 & 3 & 3 & 1 & 1 & 2 & 2 & 2 & 2 & 2 & 1 & - \\
\hline
\end{tabular}

1 non-reactive binary systems.

2 binary systems involving formaldehyde species and other components except carboxylic acids.

3 binary systems involving carboxylic acids.

[13] formaldehyde - methanol - water reactive system formerly identified.

(8), $\phi_{i}^{V, S}$, which accounts for the presence of the dimerization equilibria:

$y_{i} \frac{\phi_{i}^{V}(T, P, y)}{\phi_{i}^{V, S}\left(T, P_{i}^{S}(T)\right)} P=x_{i} \gamma_{i}(T, x) P_{i}^{S}(T)$

At equilibrium, components present in the vapor phase follow the perfect gas law so $\phi_{i}^{V}(T, P, y)=1$. The correction terms, also termed vapor fugacity coefficients of pure constituant i at saturation pressure, are calculated as follows: $\phi_{i}^{V, S}=\frac{-1+\sqrt{1+4 K(T) P_{i}^{S}(T)}}{2 K(T) P_{i}^{S}(T)}$. Note that $\phi_{i}^{V, S}\left(T, P_{i}^{S}(T)\right)=1$ for all other components.

Chemical reaction equilibrium constants are obtained as follows:

- Direct dimerization of carboxylic acid A: $\log K_{A_{2}}=\alpha_{A}+\frac{\beta_{A}}{T}$

- Direct dimerization of carboxylic acid B: $\log K_{B_{2}}=\alpha_{B}+\frac{\beta_{B}}{T}$

- Crossed dimerization of carboxylic acid A and carboxylic acid B:

$K_{A B}=\sqrt{K_{A} K_{B}}$ and $\log K_{A B}=\alpha_{A B}+\frac{\beta_{A B}}{T}$

For direct dimerization, parameters $\alpha_{A}, \alpha_{B}, \beta_{A}, \beta_{B}$ are taken from the DECHEMA literature [24]:

\begin{tabular}{lll}
\hline Component & á & $\hat{a}$ \\
\hline Acetic acid & -10.421 & 3166 \\
Formic acid & -10.743 & 3083 \\
Propionic acid & -10.843 & 3316 \\
\hline
\end{tabular}

When available, experimental data were used for the identification and simulated data were generated using UNIFAC Original when not available.

Table 4 summarizes the different cases considered for the identification of the UNIQUAC binary interaction parameters.

In the case of systems with formaldehyde species (with or without carboxylic acids), some preliminary calculations showed that it was not useful to differentiate between the binary interaction parameters of the poly(oxymethylene) glycols $\left(M G_{n}\right)$ and poly(oxymethylene) hemiformals $\left(H F_{n}\right)$ from degree 2 to 7 with other components. This indeed assumes that the interaction in the second term of the residual part of the activity coefficient for these

Table 4

Assumptions for the identification of UNIQUAC binary interaction parameters.

\begin{tabular}{|c|c|c|c|c|}
\hline Case $\mathrm{n \#}$ & Binaries & ã calculation & $\phi$ calculation & Chemical equilibrium constants \\
\hline 1: Non-reactive binaries & $\begin{array}{l}\text { Methanol - water, furfural - furfuryl } \\
\text { alcohol, furfural - water, furfural - } \\
\text { methanol, furfuryl alcohol - water, } \\
\text { furfuryl alcohol - methanol }\end{array}$ & UNIQUAC & Mixture of perfect gases & - \\
\hline $\begin{array}{l}\text { 2: Binaries involving formaldehyde } \\
\text { species and components which are } \\
\text { not carboxylic acids }\end{array}$ & $\begin{array}{l}\text { Furfural - } \text { fformaldehyde, MG, HF, } \\
\left.M G_{n}, H F_{n}\right\} \text {, furfuryl alcohol - } \\
\left\{\text { formaldehyde, MG, HF, } M G_{n}, H F_{n}\right\}\end{array}$ & UNIQUAC & Mixture of perfect gases & $\begin{array}{l}\text { Not taken into account in the } \\
\text { identification }\end{array}$ \\
\hline 3: Binaries involving carboxylic acids & $\begin{array}{l}\text { Acetic acid - formic acid, acetic acid - } \\
\text { propionic acid, acetic acid - furfural, } \\
\text { acetic acid - furfuryl alcohol, acetic acid } \\
\text { - methanol, acetic acid - water, acetic } \\
\text { acid - } \text { formaldehyde, MG, HF, } \\
M G_{n}, H F_{n} \text { \}, formic acid - propionic acid, } \\
\text { formic acid - furfural, formic acid - } \\
\text { furfuryl alcohol, formic acid - } \\
\text { methanol, formic acid - water, } \\
\text { propionic acid - furfural, formic acid - } \\
\text { \{formaldehyde, MG, HF, } M G_{n}, H F_{n} \text { \}, } \\
\text { propionic acid - furfuryl alcohol, } \\
\text { propionic acid - methanol, propionic } \\
\text { acid - water, propionic acid - } \\
\text { \{formaldehyde, MG, HF, } M G_{n}, H F_{n} \text { \} }\end{array}$ & UNIQUAC & $\begin{array}{l}\text { Mixture of perfect gases }+ \\
\text { Association term }\end{array}$ & $\begin{array}{l}\text { Not necessary because chemical } \\
\text { equilibrium constants are already taken } \\
\text { into account in the association term }\end{array}$ \\
\hline
\end{tabular}


Table 5

Binary interaction parameters estimated from literature data.

\begin{tabular}{|c|c|c|c|c|c|c|}
\hline Component 1 & Component 2 & $A_{i j}^{0}$ & $A_{j i}^{0}$ & $A_{i j}^{T}$ & $A_{j i}^{T}$ & References of data used for the identification \\
\hline Acetic acid & Formic acid & -174 & -173 & -0.36 & 1.50 & {$[25-27]$} \\
\hline Acetic acid & Furfural & 379 & -482 & -0.02 & 0.79 & {$[28]$} \\
\hline Acetic acid & Methanol & 130 & 1189 & 0.91 & -4.52 & {$[29,30]$} \\
\hline Acetic acid & Propionic acid & -46 & 52 & -0.05 & 0.16 & {$[31-33,25,26]$} \\
\hline Acetic acid & Water & 46 & 306 & -1.00 & 0.60 & {$[35,36,34]$} \\
\hline Formic acid & Furfural & 1904 & -316 & -6.00 & 3.90 & [37] \\
\hline Formic acid & Propionic acid & -828 & 1539 & 1.83 & -3.00 & {$[25,26,32]$} \\
\hline Formic acid & Water & -205 & -205 & -0.17 & 0.20 & [36] \\
\hline Furfural & Furfuryl alcohol & 533 & -570 & 0.05 & 0.67 & [38] \\
\hline Furfural & Methanol & 751 & 1106 & -0.93 & -3.36 & [39] \\
\hline Furfural & Water & 475 & 691 & -1.70 & 0.12 & {$[25,41]$} \\
\hline Furfuryl alcohol & Water & 118 & 82 & -1.37 & 2.05 & {$[42,43]$} \\
\hline Propionic acid & Methanol & 1156 & -101 & -0.38 & -1.33 & {$[44]$} \\
\hline Propionic acid & Water & 157 & 211 & -1.09 & 1.50 & [36] \\
\hline Methanol & Water & 156 & -369 & 0.91 & 0.20 & {$[45,46]$} \\
\hline
\end{tabular}

compounds is equal to that of the poly(oxymethylene) hemiformal and poly(oxymethylene) glycol of degree 2 for all the poly(oxymethylene) hemiformals $\left(H F_{n}\right)$ and poly(oxymethylene) glycol $\left(M G_{n}\right)$. Finally, with this simplification, 46 sets of parameters have to be identified.

The UNIQUAC binary interaction parameters were identified by minimizing the relative errors (see equations (10)-(19)) between experimental data when available, or simulated data generated using UNIFAC Original when not available, and UNIQUAC vapor-liquid equilibrium calculations:

$\min _{\tau_{i j i}^{0}, \tau_{i j}^{T}, \tau_{j i}^{0}, \tau_{j i}^{T}} F_{o b j}$
$F_{o b j}=\frac{F_{o b j, b u b b l e}+F_{o b j, d e w}}{n_{\text {bubble }}+n_{\text {dew }}}$

where for $(\mathrm{T}, \mathrm{X}, \mathrm{y})$ diagrams:

$$
\begin{aligned}
F_{\text {obj }, \text { bubble }}= & \sum_{l=1}^{n_{\text {bubble }}} \frac{\left(\left|y_{1, \text { exp }}^{\text {bubble }}-y_{1, \text { calc }}^{\text {bubble }}\right|\right)_{l}}{\left(\bar{y}_{1}^{\text {bubble }}\right)_{l}}+\frac{\left(\left|y_{2, \text { exp }}^{\text {bubble }}-y_{2, \text { calc }}^{\text {bubble }}\right|\right)_{l}}{\left(\bar{y}_{2}^{\text {bubble }}\right)_{l}} \\
& +\frac{\left(\left|P_{\text {exp }}^{\text {bubble }}-P_{\text {calc }}^{\text {bubble }}\right|\right)_{l}}{\left(\bar{P}^{\text {bubble }}\right)_{l}}
\end{aligned}
$$

Table 6

\begin{tabular}{|c|c|c|c|c|c|}
\hline Component 1 & Component 2 & $A_{i j}^{0}$ & $A_{j i}^{0}$ & $A_{i j}^{T}$ & $A_{j i}^{T}$ \\
\hline Formaldehyde & Acetic acid & 396 & -613 & -0.95 & 1.10 \\
\hline Formaldehyde & Formic acid & 290 & -499 & 0.89 & 0.10 \\
\hline Formaldehyde & Furfural & 102 & -107 & -0.60 & 0.20 \\
\hline Formaldehyde & Furfuryl alcohol & 51 & 89 & -0.14 & -0.79 \\
\hline Formaldehyde & Propionic acid & 372 & -572 & -1.00 & 1.10 \\
\hline Methylene Glycol (MG) & Acetic acid & -29 & 375 & -1.29 & 2.00 \\
\hline Methylene glycol (MG) & Formic acid & -78 & 74 & 0.14 & -0.90 \\
\hline Methylene glycol (MG) & Furfural & -218 & 836 & -1.44 & 1.50 \\
\hline Methylene glycol (MG) & Furfuryl alcohol & -545 & 359 & -0.24 & -0.47 \\
\hline Methylene glycol (MG) & Propionic acid & -100 & 615 & -0.74 & 2.00 \\
\hline Poly(oxymethylene) glycols $\left(M G_{i, i \geq 2}\right)$ & Acetic acid & -733 & 936 & -0.01 & -1.50 \\
\hline Poly(oxymethylene) glycols $\left(M G_{i, i \geq 2}\right)$ & Formic acid & -474 & -46 & -0.38 & -0.12 \\
\hline Poly(oxymethylene) glycols $\left(M G_{i, i \geq 2}\right)$ & Furfural & 470 & -126 & 0.62 & -1.50 \\
\hline Poly(oxymethylene) glycols $\left(M G_{i, i \geq 2}\right)$ & Furfuryl alcohol & 664 & -195 & 0.34 & -1.49 \\
\hline Poly(oxymethylene) glycols $\left(M G_{i, i \geq 2}\right)$ & Propionic acid & 30 & -136 & 0.47 & -0.83 \\
\hline Hemiformal (HF) & Acetic acid & 16 & -22 & 0.54 & -1.03 \\
\hline Hemiformal (HF) & Formic acid & 14 & -29 & -0.07 & -0.73 \\
\hline Hemiformal (HF) & Furfural & 91 & -75 & 0.66 & -1.50 \\
\hline Hemiformal (HF) & Furfuryl alcohol & 103 & -85 & 0.97 & -1.50 \\
\hline Hemiformal (HF) & Propionic acid & 470 & -309 & -0.24 & -0.24 \\
\hline Poly(oxymethylene) hemiformals $\left(H F_{i, i \geq 2}\right)$ & Acetic acid & -355 & 32 & -0.20 & -0.27 \\
\hline Poly(oxymethylene) hemiformals $\left(H F_{i, i \geq 2}\right)$ & Formic acid & -468 & -13 & -0.21 & -0.22 \\
\hline Poly(oxymethylene) hemiformals $\left(H F_{i, i \geq 2}\right)$ & Furfural & 290 & -116 & 0.37 & -1.48 \\
\hline Poly(oxymethylene) hemiformals $\left(H F_{i, i \geq 2}\right)$ & Furfuryl alcohol & 1207 & -454 & -1.11 & -0.87 \\
\hline Poly(oxymethylene) hemiformals $\left(H F_{i, i \geq 2}\right)$ & Propionic acid & -54 & 51 & -0.15 & -0.59 \\
\hline Acetic acid & Furfuryl alcohol & 435 & -374 & 0.13 & -0.12 \\
\hline Formic acid & Furfuryl alcohol & 107 & -175 & 1.00 & -0.30 \\
\hline Formic acid & Methanol & -402 & 675 & 0.00 & 0.00 \\
\hline Furfural & Propionic acid & -414 & 883 & -0.04 & -0.34 \\
\hline Furfuryl alcohol & Methanol & 440 & -388 & -0.46 & 0.54 \\
\hline Furfuryl alcohol & Propionic acid & 435 & -374 & 0.13 & -0.12 \\
\hline
\end{tabular}

Binary interaction parameters estimated from UNIFAC. 


$$
\begin{aligned}
F_{\text {obj,dew }}= & \sum_{l=1}^{n_{\text {dew }}} \frac{\left(\left|x_{1, \text { exp }}^{\text {dew }}-x_{1, \text { calc }}^{\text {dew }}\right|\right)_{l}}{\left(\bar{x}_{1}^{\text {dew }}\right)_{l}}+\frac{\left(\left|x_{2, \text { exp }}^{\text {dew }}-x_{2, \text { calc }}^{\text {dew }}\right|\right)_{l}}{\left(\bar{x}_{2}^{\text {dew }}\right)_{l}} \\
& +\frac{\left(\left|P_{\text {exp }}^{\text {dew }}-P_{\text {calc }}^{\text {dew }}\right|\right)_{l}}{\left(\bar{P}^{\text {dew }}\right)_{l}}
\end{aligned}
$$

and for $(\mathrm{P}, \mathrm{X}, \mathrm{y})$ diagrams:

$$
\begin{aligned}
F_{\text {obj,bubble }}= & \sum_{l=1}^{n_{\text {bubble }}} \frac{\left(\left|y_{1, \text { exp }}^{\text {bubble }}-y_{1, \text { calc }}^{\text {bubble }}\right|\right)_{l}}{\left(\bar{y}_{1}^{\text {bubble }}\right)_{l}}+\frac{\left(\left|y_{2, \text { exp }}^{\text {bubble }}-y_{2, \text { calc }}^{\text {bubble }}\right|\right)_{l}}{\left(\bar{y}_{2}^{\text {bubble }}\right)_{l}} \\
& +\frac{\left(\left|T_{\text {exp }}^{\text {bubble }}-T_{\text {calc }}^{\text {buble }}\right|\right)_{l}}{\left(\bar{T}^{\text {bubble }}\right)_{l}}
\end{aligned}
$$

$$
\begin{aligned}
F_{\text {obj,dew }}= & \sum_{l=1}^{n_{\text {dew }}} \frac{\left(\left|x_{1, \text { exp }}^{\text {dew }}-x_{1, \text { calc }}^{\text {dew }}\right|\right)_{l}}{\left(\bar{x}_{1}^{\text {dew }}\right)_{l}}+\frac{\left(\left|x_{2, \text { exp }}^{\text {dew }}-x_{2, \text { calc }}^{\text {dew }}\right|\right)_{l}}{\left(\bar{x}_{2}^{\text {dew }}\right)_{l}} \\
& +\frac{\left(\left|T_{\text {exp }}^{\text {dew }}-T_{\text {calc }}^{\text {dew }}\right|\right)_{l}}{\left(\bar{T}^{\text {dew }}\right)_{l}}
\end{aligned}
$$

with

$$
\begin{aligned}
\left(\bar{y}_{k}^{\text {index }}\right)_{l} & =\frac{y_{k, \text { exp }}^{\text {index }}+y_{k, \text { calc }}^{\text {index }}}{2} \text { with } k \in[1 ; 2] \text { and index } \\
& =\text { bubble or dew } \\
\left(\bar{x}_{k}^{\text {index }}\right)_{l} & =\frac{x_{k, e x p}^{\text {index }}+x_{k, \text { calc }}^{\text {index }}}{2} \text { with } k \in[1 ; 2] \text { and index } \\
& =\text { bubble or dew } \\
\left(\bar{P}^{\text {index }}\right)_{l} & =\frac{P_{\text {exp }}^{\text {index }}+P_{\text {calc }}^{\text {index }}}{2} \text { with index }=\text { bubble or dew } \\
\left(\bar{T}^{\text {index }}\right)_{l} & =\frac{T_{\text {exp }}^{\text {index }}+T_{\text {calc }}^{\text {index }}}{2} \text { with index }=\text { bubble or dew }
\end{aligned}
$$

\begin{tabular}{|c|c|c|c|c|c|}
\hline Compound 1 & Compound 2 & Type of diagram & $\overline{\Delta T}$ or $\overline{\Delta P}(\%)$ & $\overline{\Delta y_{1}}(\%)$ & References \\
\hline Formic acid & Acetic acid & $\mathrm{T}=70^{\circ} \mathrm{C}$ & 2.38 & 2.08 & {$[25]$} \\
\hline Formic acid & Acetic acid & $\mathrm{T}=30^{\circ} \mathrm{C}$ & 4.37 & 3.52 & [26] \\
\hline Formic acid & Acetic acid & $\mathrm{P}=1013 \mathrm{mbar}$ & 0.21 & 3.05 & [27] \\
\hline Acetic acid & Furfural & $\mathrm{P}=493 \mathrm{mbar}$ & 0.77 & 1.22 & [28] \\
\hline Acetic acid & Furfural & $\mathrm{P}=890 \mathrm{mbar}$ & 0.39 & 0.95 & [28] \\
\hline Acetic acid & Methanol & $\mathrm{P}=1013 \mathrm{mbar}$ & 3.24 & 4.86 & [30] \\
\hline Acetic acid & Methanol & $\mathrm{P}=941 \mathrm{mbar}$ & 1.00 & 4.23 & [29] \\
\hline Acetic acid & Propionic acid & $\mathrm{T}=70^{\circ} \mathrm{C}$ & 2.03 & 0.51 & [25] \\
\hline Acetic acid & Propionic acid & $\mathrm{T}=40^{\circ} \mathrm{C}$ & 1.00 & 0.87 & [33] \\
\hline Acetic acid & Propionic acid & $\mathrm{T}=30^{\circ} \mathrm{C}$ & 8.07 & 10.39 & [26] \\
\hline Acetic acid & Propionic acid & $\mathrm{P}=1000 \mathrm{mbar}$ & 0.04 & 2.59 & [32] \\
\hline Acetic acid & Propionic acid & $\mathrm{P}=1013 \mathrm{mbar}$ & 3.51 & 1.69 & [31] \\
\hline Water & Acetic acid & $\mathrm{P}=1013 \mathrm{mbar}$ & 0.22 & 1.07 & [34] \\
\hline Water & Acetic acid & $\mathrm{P}=167$ mbar & 0.61 & 0.50 & [35] \\
\hline Water & Acetic acid & $\mathrm{P}=333$ mbar & 0.11 & 0.41 & [35] \\
\hline Water & Acetic acid & $\mathrm{P}=93 \mathrm{mbar}$ & 0.78 & 8.88 & [36] \\
\hline Formic acid & Furfural & $\mathrm{P}=1013 \mathrm{mbar}$ & 0.57 & 8.10 & [37] \\
\hline Formic acid & Propionic acid & $\mathrm{T}=70^{\circ} \mathrm{C}$ & 2.09 & 1.68 & [25] \\
\hline Formic acid & Propionic acid & $\mathrm{T}=30^{\circ} \mathrm{C}$ & 2.76 & 6.98 & [26] \\
\hline Formic acid & Propionic acid & $\mathrm{P}=1000 \mathrm{mbar}$ & 0.34 & 3.92 & [32] \\
\hline Formic acid & Propionic acid & $\mathrm{P}=1013 \mathrm{mbar}$ & 0.33 & - & [31] \\
\hline Water & Formic acid & $\mathrm{P}=1013 \mathrm{mbar}$ & 0.12 & 1.01 & [36] \\
\hline Water & Formic acid & $\mathrm{P}=266 \mathrm{mbar}$ & 0.25 & 3.48 & [36] \\
\hline Water & Formic acid & $\mathrm{P}=93 \mathrm{mbar}$ & 0.66 & 3.67 & [36] \\
\hline Furfural & Furfuryl alcohol & $\mathrm{P}=33 \mathrm{mbar}$ & 0.54 & 8.73 & [38] \\
\hline Methanol & Furfural & $\mathrm{P}=400 \mathrm{mbar}$ & - & 11.76 & [39] \\
\hline Water & Furfural & $\mathrm{P}=1013 \mathrm{mbar}$ & 1.00 & 2.34 & [40] \\
\hline Water & Furfural & $\mathrm{P}=946 \mathrm{mbar}$ & 1.12 & 1.02 & [41] \\
\hline Water & Furfuryl alcohol & $\mathrm{P}=40 \mathrm{mbar}$ & 7.39 & 0.75 & [43] \\
\hline Water & Furfuryl alcohol & $\mathrm{P}=73 \mathrm{mbar}$ & 3.08 & 0.82 & [42] \\
\hline Propionic acid & Methanol & $\mathrm{T}=25^{\circ} \mathrm{C}$ & 1.53 & - & [44] \\
\hline Propionic acid & Methanol & $\mathrm{T}=27^{\circ} \mathrm{C}$ & 1.67 & - & [44] \\
\hline Propionic acid & Methanol & $\mathrm{T}=35^{\circ} \mathrm{C}$ & 1.63 & - & [44] \\
\hline Propionic acid & Methanol & $\mathrm{T}=45^{\circ} \mathrm{C}$ & 1.20 & - & [44] \\
\hline Propionic acid & Water & $\mathrm{P}=1013 \mathrm{mbar}$ & 0.83 & 1.98 & [36] \\
\hline Propionic acid & Water & $\mathrm{P}=266 \mathrm{mbar}$ & 0.68 & 1.41 & [36] \\
\hline Propionic acid & Water & $\mathrm{P}=93 \mathrm{mbar}$ & 0.73 & 1.29 & [36] \\
\hline Water & Methanol & $\mathrm{P}=1013 \mathrm{mbar}$ & 0.18 & 1.90 & [45] \\
\hline Water & Methanol & $\mathrm{P}=666 \mathrm{mbar}$ & 0.43 & 3.58 & [46] \\
\hline Water & Methanol & $\mathrm{P}=466 \mathrm{mbar}$ & 0.84 & 5.46 & [46] \\
\hline Water & Methanol & $\mathrm{P}=266 \mathrm{mbar}$ & 0.57 & 6.37 & [46] \\
\hline
\end{tabular}

The identification was performed using the Excel solver which

Table 7

Average deviation of the gas-phase composition and average deviation of the pressure or temperature for binary vapor-liquid and chemical equilibria plotted in Figs. 2 and 3. 
provides a multidimensional constrained non-linear method of minimization (non-linear GRG method), coupled with the Simulis Thermodynamics add-in for estimation of thermodynamic properties.

\section{Results and discussions}

\subsection{UNIQUAC binary interaction parameters estimation}

The UNIQUAC binary interaction parameters estimated in this work are reported in Tables 4 and 5. For all data points used in this work, the mean relative error was calculated as $F_{o b j}=5.60 \%$ with

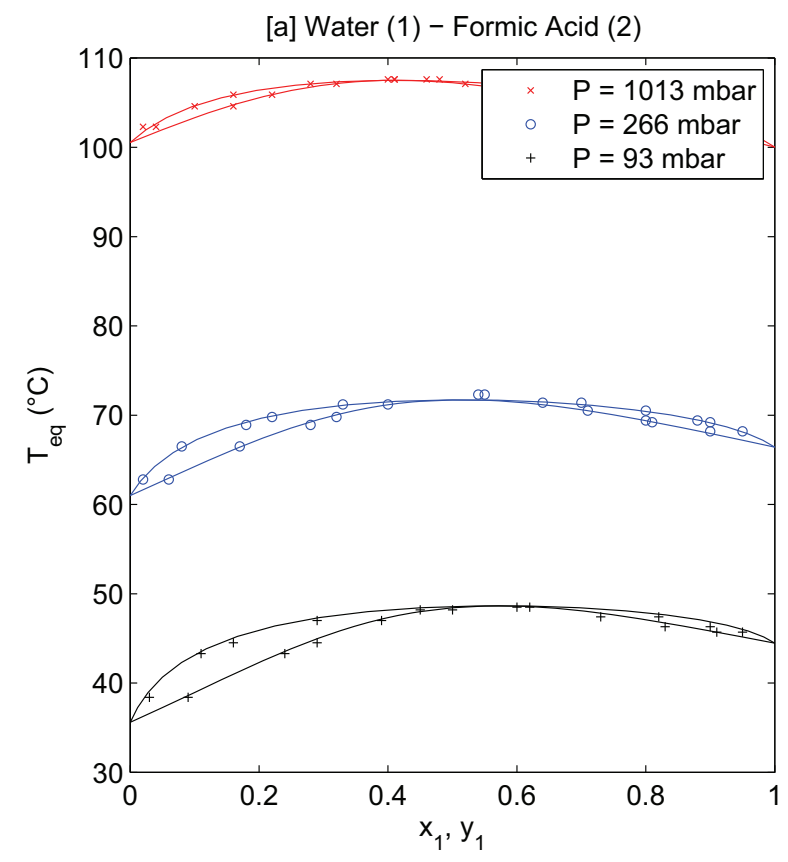

[c] Water(1) - Propionic Acid (2)

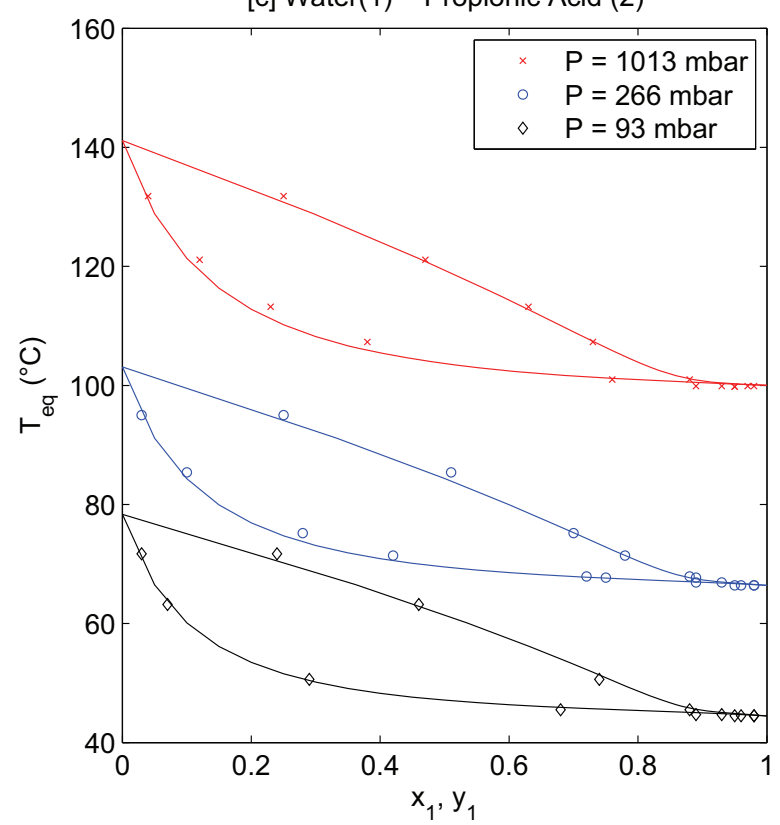

$n_{\exp }=671$. This relatively low value indicates a good estimation of the UNIQUAC binary interaction parameters as can be seen from the graphical comparisons of experimental data and calculated data presented in the Section 4.2.

Table 5 reports the values of the estimated UNIQUAC binary interaction parameters for which literature vapor-liquid data were available. Table 6 reports the binary interaction parameters of binaries for which no experimental data were available in literature. Excluding systems with formaldehyde species, few binaries are concerned. Nonetheless, interactions between formaldehyde species and other components like carboxylic acids were not studied in the literature.
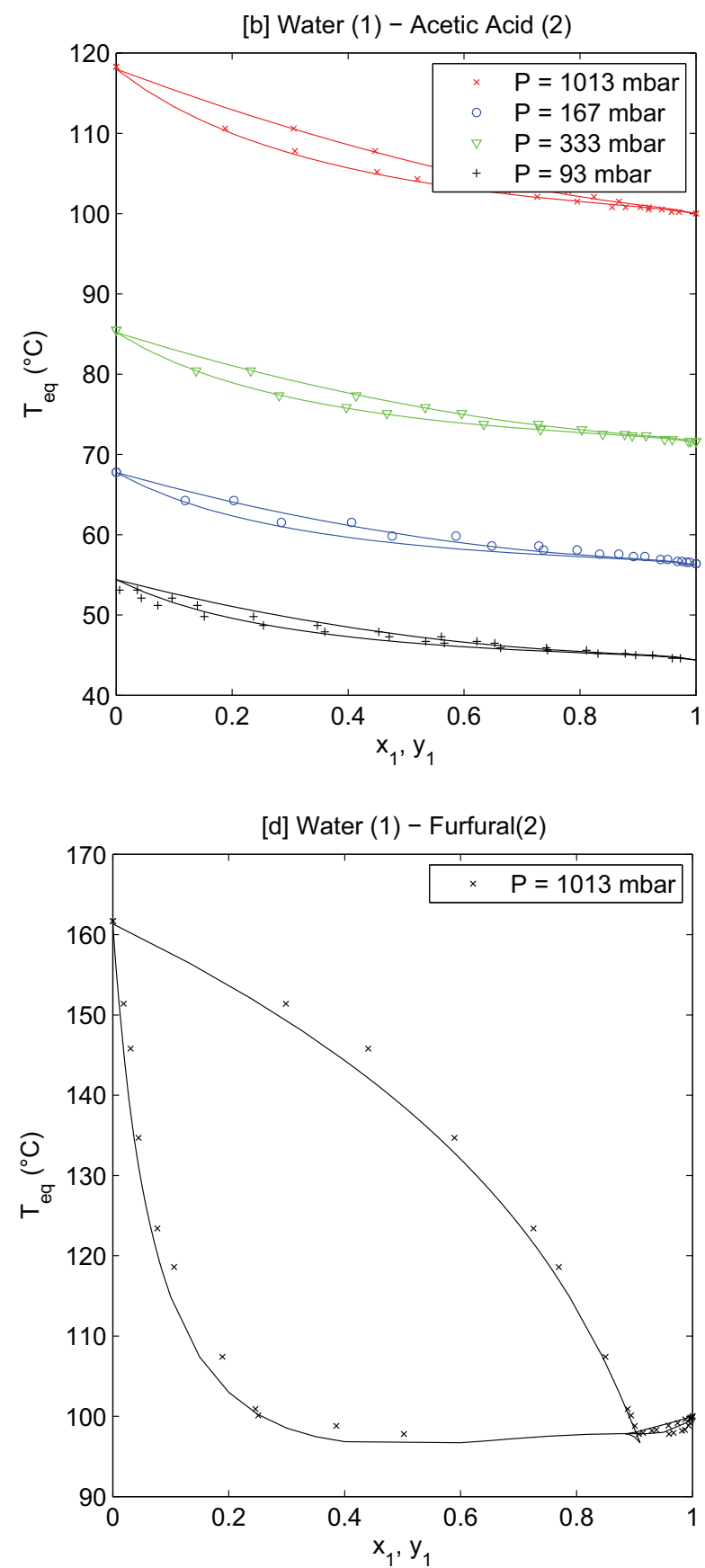

Fig. 2. Prediction of azeotropic systems vapor-liquid and chemical equilibria at different pressures and temperatures. $(x)$ experimental data from the literature. Solid line: predicted phase diagram with UNIQUAC model coupled to chemical equilibria. 
5.2. Comparison of the UNIQUAC model with binary vapor-liquid data used for the identification

This section presents the comparison of each set of experimental data used for the identification with the calculated data obtained with the estimated UNIQUAC binary interaction parameters. References of the experimental data are indicated in Table 7.

For each binary system, the average deviation of the vapor composition and the average deviation of the pressure - in the case of $(\mathrm{T}, \mathrm{x}, \mathrm{y})$ diagrams - or the average deviation for the temperature in the case of $(\mathrm{P}, \mathrm{x}, \mathrm{y})$ diagrams - between experimental data and our work were calculated as:

[a] Methanol (1) - Acetic Acid (2)

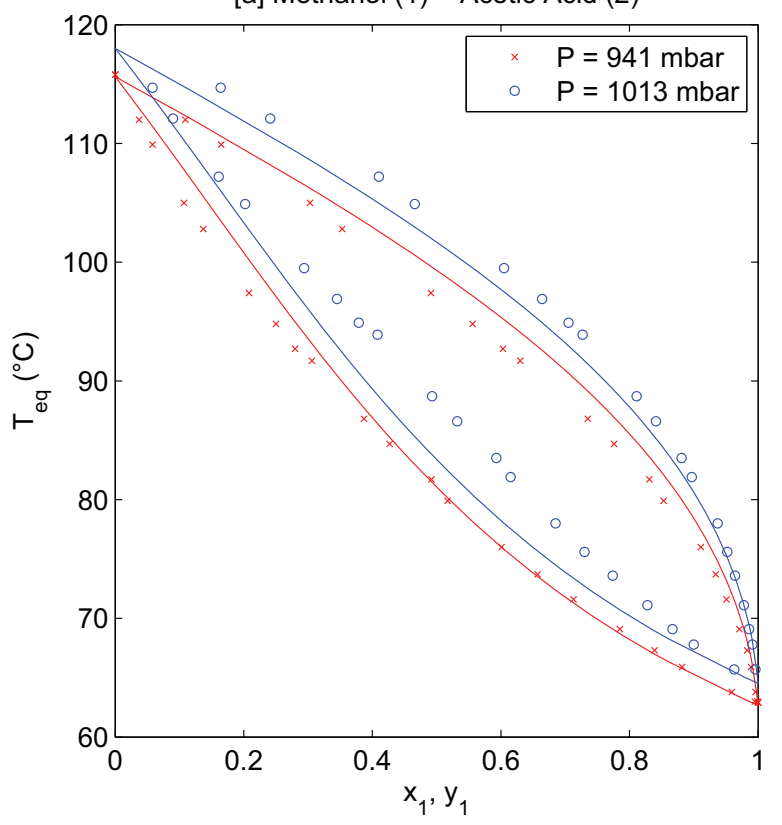

[c] Methanol (1) - Furfural (2)

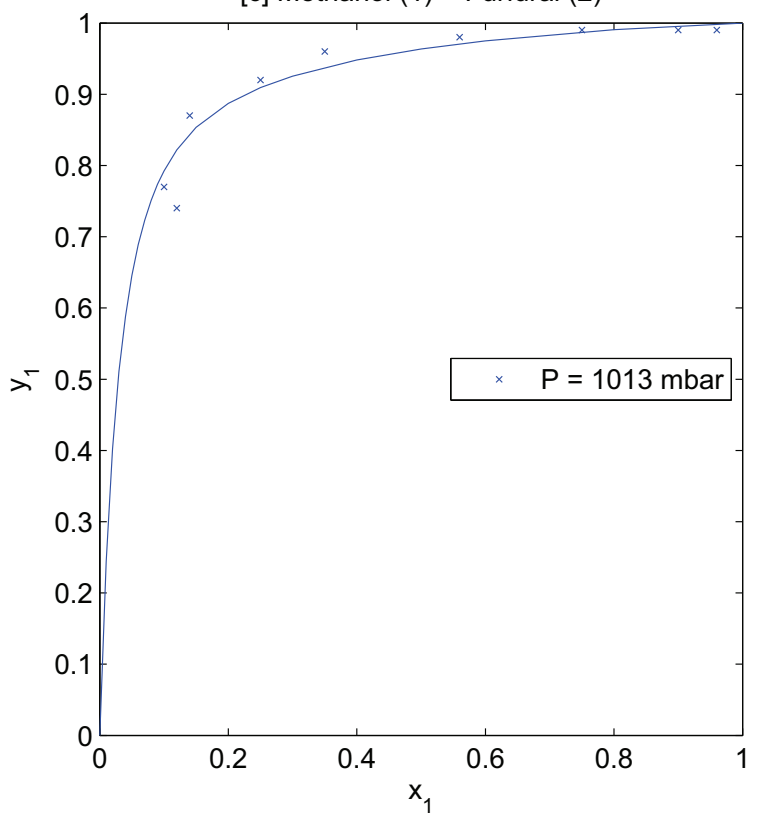

$\overline{\Delta y_{1}}=\frac{1}{n_{\exp }} \sum_{l=1}^{n_{\exp }} \frac{\left(\left|y_{1, \exp }-y_{1, \text { calc }}\right|\right)_{l}}{\overline{y_{1, l}}}$

$\overline{\Delta P}=\frac{1}{n_{\exp }} \sum_{l=1}^{n_{\text {exp }}} \frac{\left(\left|P_{\exp }-P_{\text {calc }}\right|\right)_{l}}{\bar{P}_{l}}$

$\overline{\Delta T}=\frac{1}{n_{\exp }} \sum_{l=1}^{n_{\exp }} \frac{\left(\left|T_{\exp }-T_{\text {calc }}\right|\right)_{l}}{\overline{T_{l}}}$
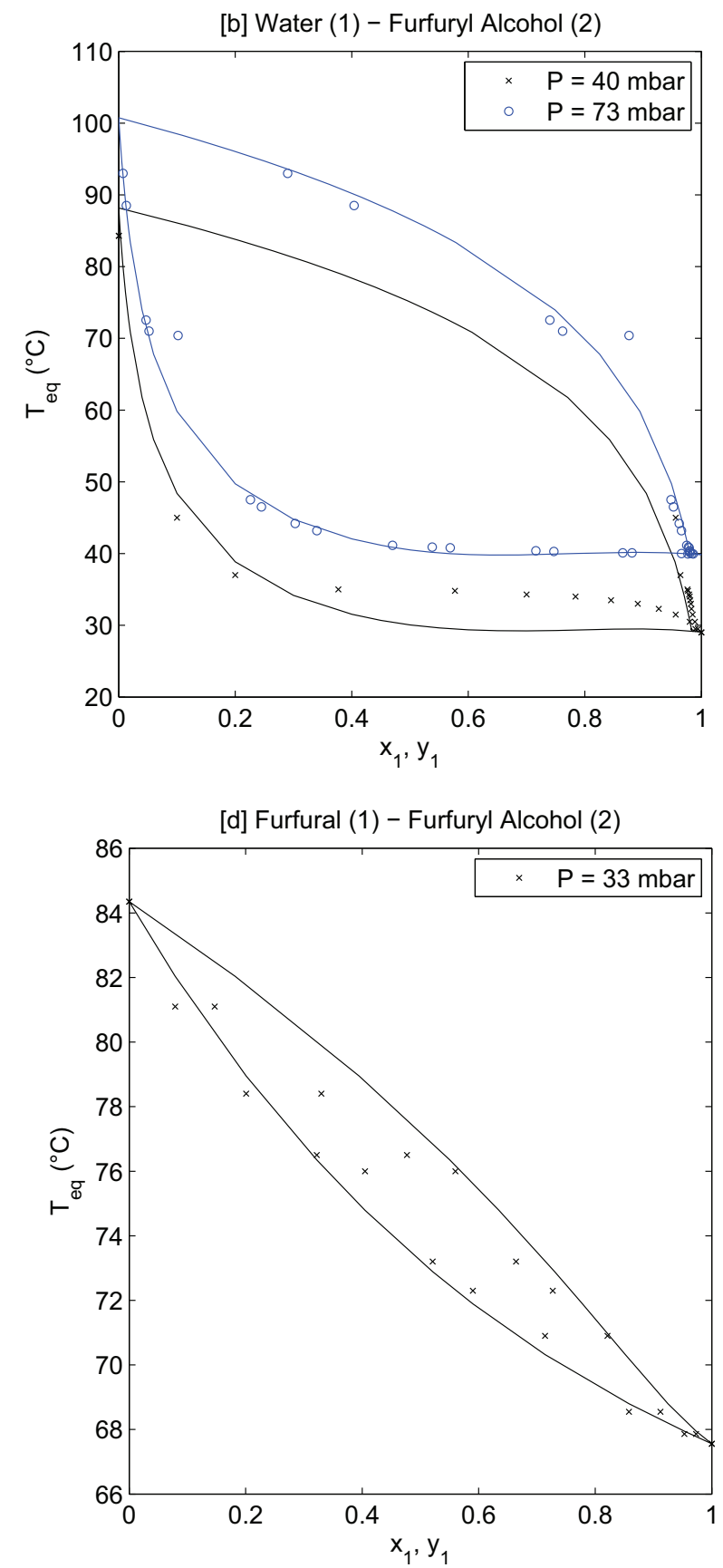

Fig. 3. Prediction of binary systems vapor-liquid and chemical equilibria at different pressures and temperatures. $(x)$ experimental data from the literature. Solid line: predicted phase diagram with UNIQUAC model coupled to chemical equilibria. 
with:

$\bar{y}_{1, l}=\frac{\left(y_{1, \exp }+y_{1, \text { calc }}\right)_{l}}{2}$

$\overline{P_{l}}=\frac{\left(P_{\exp }+P_{\text {calc }}\right)_{l}}{2}$

$\bar{T}_{l}=\frac{\left(T_{\exp }+T_{\text {calc }}\right)_{l}}{2}$

Table 7 presents the average deviation for the gas-phase composition and the average deviation for the equilibrium pressure (or temperature) between experimental data from the literature and the model, for all binary systems plotted in Figs. 2 and 3. Pressure and temperature deviations are between $0.04 \%$ and $8.07 \%$, and gas-phase composition deviations between $0.41 \%$ and $11.76 \%$. These values indicate that the UNIQUAC model developed in this work provides a fairly good description of the different binary systems, for large pressure and composition ranges. Note that all deviation values are very similar, which means that the quality of the prediction is similar for all binary systems.

Fig. 2 shows the isobaric diagram of the following binary systems: (a) water - formic acid; (b) water - acetic acid; (c) water propionic acid; (d) water - furfural. Good agreement was obtained between experimental data and the prediction of the azeotropic point at different pressures for all systems.

Fig. 3 presents the isobaric diagram of the following binary systems: (a) methanol - acetic acid; (b) water - furfuryl alcohol; (c) methanol - furfural; (d) furfural - furfuryl alcohol. Note that some inconsistent experimental points explain the larger deviation observed between the model and the experimental data.

Globally, all the figures and table confirm that estimated binary interaction coefficients give a good representation of all binaries. Every experimental data used for the identification are indeed adequately represented by the model.

\subsection{Validation of the model from comparison with ternary vapor-liquid reactive equilibria}

The complete reactive vapor-liquid model was used to validate the use of the binary interaction parameters for a ternary system. The model includes equations for the vapor-liquid equilibrium

Table 8

Chemical reaction equilibrium constants: $\ln K=a_{1}+a_{2} / T$.

\begin{tabular}{|c|c|c|c|c|c|}
\hline Reaction & Phase & Heat of reaction $(\mathrm{kJ} / \mathrm{mol})$ & $a_{1}$ & $a_{2}$ & References \\
\hline$W+F A \Leftrightarrow M G$ & Vapor & -43.51 & -16.984 & 5233.2 & {$[50]$} \\
\hline $\begin{array}{l}2 M G \Leftrightarrow M G_{2}+W \\
M G+M G_{2} \Leftrightarrow M G_{3}+W \\
M G+M G_{3} \Leftrightarrow M G_{4}+W\end{array}$ & Liquid & -0.234 & $4.98 .10^{-3}$ & 869.5 & {$[51,20]$} \\
\hline $\begin{array}{l}M G+M G_{4} \Leftrightarrow M G_{5}+W \\
M G+M G_{5} \Leftrightarrow M G_{6}+W \\
M G+M G_{6} \Leftrightarrow M G_{7}+W\end{array}$ & Liquid & -0.234 & $1.908 .10^{-2}$ & 544.5 & {$[51,20]$} \\
\hline $\begin{array}{l}M E+F A \Leftrightarrow H F \\
2 H F \Leftrightarrow H F_{2}+M E \\
H F+H F_{2} \Leftrightarrow H F_{3}+M E\end{array}$ & Vapor & -53.73 & -14.755 & 5969.4 & [49] \\
\hline $\begin{array}{l}H F+H F_{3} \Leftrightarrow H F_{4}+M E \\
H F+H F_{4} \Leftrightarrow H F_{5}+M E \\
H F+H F_{5} \Leftrightarrow H F_{6}+M E \\
H F+H F_{6} \Leftrightarrow H F_{7}+M E\end{array}$ & Liquid & -7.00 & -0.4966 & -491.3 & [49] \\
\hline
\end{tabular}

Table 9

Average deviation of the gas-phase composition and average deviation of the temperature for ternary vapor-liquid and chemical equilibria illustrated in Tables $10-13$

\begin{tabular}{|c|c|c|c|c|c|c|c|}
\hline Compound 1 & Compound 2 & Compound 3 & $P($ mbar $)$ & $\overline{\Delta T}(\%)$ & $\overline{\Delta y_{1}}(\%)$ & $\overline{\Delta y_{2}}(\%)$ & References \\
\hline Water & Acetic acid & Propionic acid & 1013 & 0.53 & 4.29 & 6.05 & [53] \\
\hline Water & Formic acid & Acetic acid & 67 & 1.44 & 2.74 & 4.61 & [54] \\
\hline Water & Formic acid & Acetic acid & 1013 & 0.22 & 2.63 & 4.51 & [55] \\
\hline Methanol & Water & Acetic acid & 1013 & 1.03 & 4.19 & 5.19 & [52] \\
\hline Water & Methanol & Furfural & 1007 & - & 9.38 & 9.14 & [56] \\
\hline Water & Methanol & Furfural & 400 & - & 7.47 & 9.59 & [56] \\
\hline
\end{tabular}

Table 10

Prediction of vapor-liquid reactive equilibria of water (1) - acetic acid (2) - propionic acid (3) ternary system [53].

\begin{tabular}{|c|c|c|c|c|c|c|c|c|c|c|c|}
\hline$P($ mbar $)$ & $x_{1}$ & $x_{2}$ & $T_{\text {eq,exp }}\left({ }^{\circ} \mathrm{C}\right)$ & $T_{\text {eq,calc }}\left({ }^{\circ} \mathrm{C}\right)$ & $\Delta T(\%)$ & $y_{1, \exp }$ & $y_{1, \text { calc }}$ & $\Delta y_{1}(\%)$ & $y_{2, \exp }$ & $y_{2, \text { calc }}$ & $\Delta y_{2}(\%)$ \\
\hline 1013 & 0.20 & 0.16 & 114.0 & 112.7 & 1.18 & 0.505 & 0.557 & 9.72 & 0.141 & 0.125 & 11.94 \\
\hline 1013 & 0.63 & 0.07 & 102.3 & 102.4 & 0.08 & 0.825 & 0.821 & 0.46 & 0.038 & 0.041 & 6.54 \\
\hline 1013 & 0.80 & 0.04 & 100.4 & 101.1 & 0.65 & 0.876 & 0.881 & 0.58 & 0.024 & 0.024 & 1.05 \\
\hline 1013 & 0.20 & 0.32 & 113.0 & 112.3 & 0.63 & 0.438 & 0.490 & 11.28 & 0.282 & 0.257 & 9.35 \\
\hline 1013 & 0.20 & 0.48 & 112.2 & 111.7 & 0.44 & 0.391 & 0.431 & 9.70 & 0.420 & 0.392 & 6.92 \\
\hline 1013 & 0.60 & 0.24 & 102.7 & 103.0 & 0.26 & 0.762 & 0.764 & 0.32 & 0.151 & 0.149 & 1.06 \\
\hline 1013 & 0.80 & 0.12 & 100.5 & 101.3 & 0.77 & 0.872 & 0.868 & 0.45 & 0.074 & 0.078 & 5.40 \\
\hline 1013 & 0.2 & 0.64 & 111.3 & 110.9 & 0.33 & 0.358 & 0.378 & 5.48 & 0.537 & 0.529 & 1.50 \\
\hline 1013 & 0.40 & 0.48 & 106.4 & 105.9 & 0.50 & 0.567 & 0.594 & 4.71 & 0.353 & 0.339 & 3.91 \\
\hline 1013 & 0.71 & 0.23 & 101.8 & 102.0 & 0.21 & 0.817 & 0.811 & 0.73 & 0.143 & 0.152 & 6.27 \\
\hline 1013 & 0.80 & 0.16 & 101.1 & 101.4 & 0.28 & 0.867 & 0.862 & 0.52 & 0.102 & 0.109 & 6.59 \\
\hline
\end{tabular}


Table 11

Prediction of vapor-liquid reactive equilibria of methanol (1) - water (2) - acetic acid (3) ternary system [52].

\begin{tabular}{|c|c|c|c|c|c|c|c|c|c|c|c|}
\hline$P($ mbar $)$ & $x_{1}$ & $x_{2}$ & $T_{\text {eq,exp }}\left({ }^{\circ} \mathrm{C}\right)$ & $T_{\text {eq,calc }}\left({ }^{\circ} \mathrm{C}\right)$ & $\Delta T(\%)$ & $y_{1, \exp }$ & $y_{1, \text { calc }}$ & $\Delta y_{1}(\%)$ & $y_{2, \text { exp }}$ & $y_{2, \text { calc }}$ & $\Delta y_{2}(\%)$ \\
\hline 1013 & 0.1148 & 0.6056 & 93.4 & 93.7 & 0.4 & 0.3442 & 0.3316 & 3.74 & 0.5361 & 0.5480 & 2.20 \\
\hline 1013 & 0.0344 & 0.6787 & 99.6 & 99.3 & 0.3 & 0.1312 & 0.1203 & 8.66 & 0.7030 & 0.7077 & 0.67 \\
\hline 1013 & 0.3555 & 0.5899 & 79.0 & 77.8 & 1.5 & 0.7039 & 0.7051 & 0.17 & 0.2682 & 0.2889 & 7.43 \\
\hline 1013 & 0.3362 & 0.5591 & 80.6 & 79.7 & 1.1 & 0.7079 & 0.6801 & 4.01 & 0.2790 & 0.3051 & 8.93 \\
\hline 1013 & 0.3795 & 0.5258 & 79.4 & 78.1 & 1.7 & 0.7237 & 0.7153 & 1.17 & 0.2608 & 0.2734 & 4.70 \\
\hline 1013 & 0.1641 & 0.7961 & 85.3 & 85.2 & 0.1 & 0.5407 & 0.5142 & 5.03 & 0.4492 & 0.4771 & 6.01 \\
\hline 1013 & 0.4069 & 0.5223 & 78.6 & 76.7 & 2.4 & 0.7530 & 0.7373 & 2.10 & 0.2394 & 0.2555 & 6.49 \\
\hline 1013 & 0.0264 & 0.3894 & 104.6 & 103.9 & 0.7 & 0.0776 & 0.0731 & 5.96 & 0.5010 & 0.5223 & 4.16 \\
\hline 1013 & 0.1268 & 0.5316 & 94.3 & 94.2 & 0.2 & 0.3741 & 0.3424 & 8.84 & 0.4867 & 0.5077 & 4.23 \\
\hline 1013 & 0.0399 & 0.6684 & 98.9 & 98.9 & 0.0 & 0.1515 & 0.1367 & 10.24 & 0.6897 & 0.6923 & 0.38 \\
\hline 1013 & 0.4386 & 0.4855 & 77.4 & 76.0 & 1.7 & 0.7765 & 0.7568 & 2.57 & 0.2157 & 0.2359 & 8.96 \\
\hline 1013 & 0.4177 & 0.4968 & 78.5 & 76.8 & 2.2 & 0.7376 & 0.7427 & 0.69 & 0.2506 & 0.2484 & 0.88 \\
\hline 1013 & 0.0467 & 0.6815 & 98.3 & 98.2 & 0.1 & 0.1786 & 0.1608 & 10.50 & 0.6782 & 0.6857 & 1.09 \\
\hline 1013 & 0.3395 & 0.5184 & 81.4 & 80.5 & 1.1 & 0.6892 & 0.6763 & 1.90 & 0.2676 & 0.3014 & 11.89 \\
\hline 1013 & 0.2225 & 0.5762 & 87.8 & 86.5 & 1.5 & 0.5558 & 0.5370 & 3.43 & 0.3869 & 0.4102 & 5.85 \\
\hline 1013 & 0.3985 & 0.5217 & 78.7 & 77.2 & 2.0 & 0.7500 & 0.7306 & 2.62 & 0.2411 & 0.2608 & 7.85 \\
\hline 1013 & 0.0702 & 0.5801 & 97.9 & 97.6 & 0.3 & 0.2190 & 0.2115 & 3.50 & 0.5949 & 0.6015 & 1.10 \\
\hline 1013 & 0.0889 & 0.6060 & 95.7 & 95.8 & 0.1 & 0.2865 & 0.2667 & 7.16 & 0.5717 & 0.5854 & 2.37 \\
\hline 1013 & 0.3583 & 0.5865 & 78.6 & 77.7 & 1.1 & 0.7309 & 0.7069 & 3.33 & 0.2634 & 0.2870 & 8.57 \\
\hline
\end{tabular}

(see equation (26)) and the chemical reaction equilibrium (see equations (30)-(33)).

Values of the chemical reaction equilibrium constants were taken from the literature $[24,47-49]$.

$$
\left\{\begin{array}{l}
\phi_{i}^{V, S}\left(T, P_{i}^{S}(T)\right)=\frac{-1+\sqrt{1+4 K(T) P_{i}^{S}(T)}}{2 K(T) P_{i}^{S}(T)} \text { for carboxylic acids } \\
\phi_{i}^{V, S}\left(T, P_{i}^{S}(T)\right)=1 \text { otherwise }
\end{array}\right.
$$

$y_{i} P=x_{i} \gamma_{i}(T, x) P_{i}^{S}(T) \phi_{i}^{V, S}\left(T, P_{i}^{S}(T)\right)$

$$
\log K(T)=a_{1}+\frac{a_{2}}{T}
$$

$\ln P_{i}^{S}(T)=a_{1}+\frac{a_{2}}{T}+a_{3} \ln T+a_{4} T^{a_{5}}$

$$
K_{M G}=\frac{y_{M G}}{y_{F A} y_{W}} \cdot \frac{P_{r e f}}{P}
$$

\begin{tabular}{|c|c|c|c|c|c|c|c|c|c|c|c|}
\hline$P($ mbar $)$ & $x_{1}$ & $x_{2}$ & $T_{\text {eq,exp }}\left({ }^{\circ} \mathrm{C}\right)$ & $T_{\text {eq,calc }}\left({ }^{\circ} \mathrm{C}\right)$ & $\Delta T(\%)$ & $y_{1, \exp }$ & $y_{1, \text { calc }}$ & $\Delta y_{1}(\%)$ & $y_{2, \exp }$ & $y_{2, \text { calc }}$ & $\Delta y_{2}(\%)$ \\
\hline 67 & 0.20 & 0.16 & 42.4 & 42.0 & 0.89 & 0.237 & 0.232 & 1.97 & 0.210 & 0.189 & 10.70 \\
\hline 67 & 0.40 & 0.12 & 41.3 & 41.1 & 0.60 & 0.446 & 0.464 & 3.85 & 0.119 & 0.110 & 7.44 \\
\hline 67 & 0.20 & 0.32 & 40.0 & 40.6 & 1.54 & 0.187 & 0.175 & 6.46 & 0.377 & 0.378 & 0.39 \\
\hline 67 & 0.40 & 0.24 & 41.0 & 41.1 & 0.24 & 0.405 & 0.413 & 1.85 & 0.244 & 0.239 & 2.08 \\
\hline 67 & 0.60 & 0.16 & 40.2 & 40.5 & 0.77 & 0.625 & 0.648 & 3.69 & 0.137 & 0.125 & 9.05 \\
\hline 67 & 0.80 & 0.08 & 39.0 & 39.6 & 1.49 & 0.838 & 0.848 & 1.22 & 0.047 & 0.046 & 1.25 \\
\hline 67 & 0.20 & 0.48 & 37.7 & 38.9 & 3.26 & 0.129 & 0.132 & 2.11 & 0.573 & 0.564 & 1.60 \\
\hline 67 & 0.40 & 0.36 & 40.2 & 41.0 & 1.94 & 0.354 & 0.364 & 2.75 & 0.404 & 0.382 & 5.65 \\
\hline 67 & 0.60 & 0.24 & 40.3 & 41.0 & 1.63 & 0.626 & 0.632 & 0.91 & 0.214 & 0.202 & 5.93 \\
\hline 67 & 0.20 & 0.64 & 36.2 & 37.1 & 2.54 & 0.100 & 0.099 & 1.36 & 0.755 & 0.747 & 1.06 \\
\hline 67 & 0.40 & 0.48 & 40.0 & 40.8 & 1.93 & 0.300 & 0.319 & 6.25 & 0.576 & 0.541 & 6.33 \\
\hline 67 & 0.60 & 0.32 & 40.8 & 41.5 & 1.64 & 0.615 & 0.617 & 0.40 & 0.300 & 0.289 & 3.80 \\
\hline 1013 & 0.20 & 0.16 & 109.2 & 109.3 & 0.13 & 0.250 & 0.273 & 8.79 & 0.160 & 0.171 & 6.68 \\
\hline 1013 & 0.20 & 0.32 & 107.3 & 108.4 & 1.04 & 0.228 & 0.227 & 0.38 & 0.337 & 0.341 & 1.23 \\
\hline 1013 & 0.20 & 0.64 & 106.0 & 106.4 & 0.41 & 0.150 & 0.164 & 8.82 & 0.713 & 0.681 & 4.53 \\
\hline 1013 & 0.40 & 0.12 & 106.3 & 106.2 & 0.08 & 0.500 & 0.516 & 3.17 & 0.101 & 0.099 & 2.36 \\
\hline 1013 & 0.40 & 0.24 & 106.7 & 106.6 & 0.09 & 0.454 & 0.480 & 5.54 & 0.217 & 0.209 & 3.69 \\
\hline 1013 & 0.40 & 0.36 & 106.8 & 106.9 & 0.10 & 0.445 & 0.447 & 0.46 & 0.335 & 0.330 & 1.56 \\
\hline 1013 & 0.40 & 0.48 & 107.1 & 107.2 & 0.08 & 0.393 & 0.418 & 6.23 & 0.482 & 0.461 & 4.41 \\
\hline 1013 & 0.60 & 0.08 & 103.8 & 103.7 & 0.09 & 0.690 & 0.709 & 2.65 & 0.058 & 0.051 & 12.16 \\
\hline 1013 & 0.60 & 0.16 & 104.4 & 104.3 & 0.08 & 0.690 & 0.698 & 1.14 & 0.114 & 0.109 & 4.08 \\
\hline 1013 & 0.60 & 0.24 & 105.3 & 104.9 & 0.35 & 0.690 & 0.688 & 0.26 & 0.181 & 0.174 & 4.00 \\
\hline 1013 & 0.60 & 0.32 & 105.3 & 105.6 & 0.29 & 0.690 & 0.681 & 1.37 & 0.229 & 0.245 & 6.71 \\
\hline 1013 & 0.80 & 0.04 & 101.9 & 101.8 & 0.06 & 0.860 & 0.862 & 0.23 & 0.023 & 0.021 & 9.88 \\
\hline 1013 & 0.80 & 0.08 & 102.0 & 102.2 & 0.20 & 0.866 & 0.867 & 0.08 & 0.044 & 0.043 & 2.64 \\
\hline 1013 & 0.80 & 0.12 & 102.4 & 102.6 & 0.18 & 0.872 & 0.872 & 0.01 & 0.068 & 0.066 & 3.30 \\
\hline 1013 & 0.80 & 0.16 & 102.7 & 103.0 & 0.30 & 0.876 & 0.879 & 0.30 & 0.089 & 0.089 & 0.39 \\
\hline
\end{tabular}

Table 12

Prediction of vapor-liquid reactive equilibria of water (1) - formic acid (2) - acetic acid (3) ternary system [54,55]. 
$K_{M G_{n}}=\frac{x_{M G_{n}} x_{W}}{x_{M G} x_{M G_{n-1}}} \cdot \frac{\gamma_{M G_{n}} \gamma_{W}}{\gamma_{M G} \gamma_{M G_{n-1}}} \quad$ with $n \in[2 ; 7]$

$K_{H F}=\frac{y_{H F}}{y_{F A} y_{M E}} \cdot \frac{P_{r e f}}{P}$

$K_{H F_{n}}=\frac{x_{H F_{n}} x_{M E}}{x_{H F_{n-1}} x_{H F}} \cdot \frac{\gamma_{H F_{n}} \gamma_{M E}}{\gamma_{H F_{n-1}} \gamma_{H F}} \quad$ with $n \in[2 ; 7]$

In this work, the chemical reaction constants (see Table 8) are expressed in the gas phase for the formation of methylene glycol (eq. (29)) and hemiformal (eq. (31)).

Binary vapor liquid data were used to regress the UNIQUAC binary interaction parameters and ternary vapor liquid data to validate the model and to check the extensibility of the model to multicomponent mixtures. Experimental data were taken from the DECHEMA literature in the same ranges of pressure and temperature where the model has been identified.

The reactive vapor liquid equilibrium of the ternary watermethanol-formaldehyde mixture was already validated in a former publication [13]. No data were available to validate the model on other systems.

Tables 10-13 give the corresponding values and relative errors of all isobaric diagrams of the following systems: methanol - water - acetic acid; water - formic acid - acetic acid; water - acetic acid propionic acid; methanol - water - furfural. Table 9 reports the average deviation of the equilibrium temperature and the average deviation of the gas-phase composition between experimental data from the literature ([52-56]) and our model, for ternary systems of

Table 13

Prediction of vapor-liquid reactive equilibria of methanol (1) - water (2) - furfural (3) ternary system [56].

\begin{tabular}{|c|c|c|c|c|c|c|c|c|}
\hline$P($ mbar $)$ & $x_{1}$ & $x_{2}$ & $y_{1, \exp }$ & $y_{1, \text { calc }}$ & $\Delta y_{1}(\%)$ & $y_{2, \exp }$ & $y_{2, \text { calc }}$ & $\Delta y_{2}(\%)$ \\
\hline 1007 & 0.0023 & 0.9956 & 0.0168 & 0.0161 & 4.02 & 0.9714 & 0.9686 & 0.28 \\
\hline 1007 & 0.0183 & 0.9654 & 0.1316 & 0.1019 & 25.4 & 0.8052 & 0.8296 & 2.99 \\
\hline 1007 & 0.0237 & 0.8866 & 0.1057 & 0.0959 & 9.73 & 0.8156 & 0.8049 & 1.32 \\
\hline 1007 & 0.0447 & 0.5827 & 0.1611 & 0.1334 & 18.7 & 0.7714 & 0.8073 & 4.55 \\
\hline 1007 & 0.0300 & 0.9600 & 0.1633 & 0.1640 & 0.44 & 0.8003 & 0.7921 & 1.03 \\
\hline 1007 & 0.1139 & 0.2025 & 0.5025 & 0.4192 & 18.0 & 0.4666 & 0.4998 & 6.86 \\
\hline 1007 & 0.0431 & 0.9425 & 0.2265 & 0.2136 & 5.86 & 0.7231 & 0.7359 & 1.75 \\
\hline 1007 & 0.1443 & 0.3721 & 0.4679 & 0.3959 & 16.6 & 0.4959 & 0.5550 & 11.2 \\
\hline 1007 & 0.0809 & 0.8111 & 0.3014 & 0.2630 & 13.6 & 0.6511 & 0.6688 & 2.69 \\
\hline 1007 & 0.0614 & 0.9284 & 0.3447 & 0.2859 & 18.6 & 0.6246 & 0.6805 & 8.57 \\
\hline 1007 & 0.1836 & 0.3266 & 0.5688 & 0.4830 & 16.3 & 0.4056 & 0.4717 & 15.0 \\
\hline 1007 & 0.1222 & 0.7963 & 0.4299 & 0.3703 & 14.8 & 0.5315 & 0.5741 & 7.71 \\
\hline 1007 & 0.1597 & 0.6628 & 0.4386 & 0.4021 & 8.67 & 0.5248 & 0.5541 & 5.43 \\
\hline 1007 & 0.2077 & 0.4922 & 0.5781 & 0.4752 & 19.5 & 0.3949 & 0.4885 & 21.1 \\
\hline 1007 & 0.2307 & 0.4103 & 0.5932 & 0.5230 & 12.5 & 0.3862 & 0.4418 & 13.4 \\
\hline 1007 & 0.1791 & 0.7164 & 0.5179 & 0.4587 & 12.1 & 0.4535 & 0.4991 & 9.57 \\
\hline 1007 & 0.2416 & 0.5369 & 0.5891 & 0.5227 & 11.9 & 0.3891 & 0.4447 & 13.3 \\
\hline 1007 & 0.3711 & 0.1649 & 0.7821 & 0.7816 & 0.06 & 0.2043 & 0.1884 & 8.12 \\
\hline 1007 & 0.2082 & 0.7182 & 0.5752 & 0.5176 & 10.5 & 0.4013 & 0.4467 & 10.7 \\
\hline 1007 & 0.3488 & 0.3721 & 0.7009 & 0.6631 & 5.55 & 0.2846 & 0.3116 & 9.05 \\
\hline 1007 & 0.3010 & 0.5313 & 0.6597 & 0.5995 & 9.56 & 0.3232 & 0.3733 & 14.4 \\
\hline 1007 & 0.4954 & 0.1467 & 0.8393 & 0.8526 & 1.58 & 0.1440 & 0.1264 & 12.9 \\
\hline 1007 & 0.3500 & 0.5333 & 0.6779 & 0.6559 & 3.30 & 0.3081 & 0.3217 & 4.33 \\
\hline 1007 & 0.5497 & 0.1566 & 0.8445 & 0.8649 & 2.38 & 0.1359 & 0.1174 & 14.5 \\
\hline 1007 & 0.3283 & 0.6097 & 0.7079 & 0.6537 & 7.95 & 0.2766 & 0.3259 & 16.3 \\
\hline 1007 & 0.3364 & 0.6131 & 0.6891 & 0.6664 & 3.36 & 0.2996 & 0.3155 & 5.17 \\
\hline 1007 & 0.3645 & 0.5814 & 0.7145 & 0.6870 & 3.92 & 0.2734 & 0.2962 & 7.99 \\
\hline 1007 & 0.3545 & 0.6304 & 0.7323 & 0.7035 & 4.02 & 0.2608 & 0.2888 & 10.2 \\
\hline 1007 & 0.4902 & 0.4628 & 0.7656 & 0.7731 & 0.97 & 0.2295 & 0.2168 & 5.71 \\
\hline 1007 & 0.9023 & 0.0902 & 0.9699 & 0.9601 & 1.01 & 0.0295 & 0.0389 & 27.5 \\
\hline 400 & 0.0011 & 0.9983 & 0.0074 & 0.0085 & 14.0 & 0.9897 & 0.9859 & 0.39 \\
\hline 400 & 0.0677 & 0.1470 & 0.4166 & 0.3887 & 6.92 & 0.5057 & 0.5082 & 0.49 \\
\hline 400 & 0.0687 & 0.6107 & 0.1752 & 0.2206 & 22.9 & 0.7661 & 0.7293 & 4.93 \\
\hline 400 & 0.1422 & 0.2299 & 0.5441 & 0.5216 & 4.22 & 0.4098 & 0.4275 & 4.22 \\
\hline 400 & 0.0413 & 0.9548 & 0.2758 & 0.2353 & 15.8 & 0.7106 & 0.7432 & 4.48 \\
\hline 400 & 0.0703 & 0.8125 & 0.2163 & 0.2501 & 14.4 & 0.7184 & 0.6772 & 5.91 \\
\hline 400 & 0.1867 & 0.4647 & 0.4566 & 0.4884 & 6.72 & 0.5072 & 0.4776 & 6.02 \\
\hline 400 & 0.2806 & 0.2329 & 0.6398 & 0.7092 & 10.2 & 0.3303 & 0.2617 & 23.1 \\
\hline 400 & 0.1150 & 0.8743 & 0.4504 & 0.4462 & 0.94 & 0.5237 & 0.5269 & 0.61 \\
\hline 400 & 0.1966 & 0.6213 & 0.4107 & 0.4935 & 18.3 & 0.5551 & 0.4698 & 16.6 \\
\hline 400 & 0.1209 & 0.8732 & 0.4405 & 0.4683 & 6.13 & 0.5472 & 0.5158 & 5.91 \\
\hline 400 & 0.1586 & 0.7753 & 0.4539 & 0.4705 & 3.60 & 0.5076 & 0.4814 & 5.31 \\
\hline 400 & 0.2681 & 0.4990 & 0.5412 & 0.5947 & 9.42 & 0.4256 & 0.3774 & 12.0 \\
\hline 400 & 0.3312 & 0.3927 & 0.6321 & 0.6797 & 7.25 & 0.3374 & 0.2969 & 12.7 \\
\hline 400 & 0.1910 & 0.7606 & 0.5397 & 0.5341 & 1.05 & 0.4473 & 0.4277 & 4.49 \\
\hline 400 & 0.1960 & 0.7521 & 0.5078 & 0.5386 & 5.88 & 0.4686 & 0.4235 & 10.1 \\
\hline 400 & 0.3354 & 0.4969 & 0.5939 & 0.6675 & 11.6 & 0.3798 & 0.3088 & 20.6 \\
\hline 400 & 0.2393 & 0.7539 & 0.6280 & 0.6375 & 1.51 & 0.3659 & 0.3541 & 3.28 \\
\hline 400 & 0.3137 & 0.6056 & 0.6353 & 0.6577 & 3.46 & 0.3414 & 0.3175 & 7.25 \\
\hline 400 & 0.3715 & 0.5872 & 0.7285 & 0.7203 & 1.13 & 0.2464 & 0.2636 & 6.76 \\
\hline 400 & 0.5327 & 0.3226 & 0.7737 & 0.8219 & 6.04 & 0.2095 & 0.1636 & 24.6 \\
\hline 400 & 0.4749 & 0.5098 & 0.7722 & 0.7951 & 2.92 & 0.2204 & 0.1992 & 10.1 \\
\hline 400 & 0.6550 & 0.3149 & 0.8477 & 0.8744 & 3.10 & 0.1453 & 0.1200 & 19.1 \\
\hline 400 & 0.7800 & 0.1907 & 0.9075 & 0.9243 & 1.83 & 0.0880 & 0.0713 & 20.9 \\
\hline
\end{tabular}


Tables 10-13 Note that these data were not used for the estimation of the binary interaction parameters but only to validate the approach. Temperature deviations are between $0.22 \%$ and $1.44 \%$. and gas-phase composition deviations between $2.63 \%$ and $9.59 \%$. This good agreement confirms that our model is able to represent the behavior of multicomponent systems.

As mentioned above, good agreement was obtained between experimental data set and estimated vapor-liquid equilibria with our model.

\section{Conclusion}

In this work a model to describe the thermodynamic behavior of a complex reactive mixture was developed. The model was applied to a representative mixture of the condensable fraction of the gaseous effluent from the wood torrefaction process. A model based on the local composition concept (UNIQUAC) was chosen and was coupled to chemical equilibria of this reactive mixture where 22 compounds and 14 chemical reactions are considered. With this uncoupling approach, effects of weak intermolecular interactions of the physical equilibria are differentiated from the strong intermolecular interactions involved in the chemical reactions. In the present case, chemical equilibrium constants were available in the literature and can then be considered as known. The hypothesis of similar interactions for poly(oxymethylene) glycols with other compounds and for poly(oxymethylene) hemiformals with other compounds allowed to limit to 46 the number of unknown vapor-liquid equilibrium binary interaction parameters. They were identified here from litterature data for binary systems when available or from simulated data when not. This approach was validated by comparison with available experimental data for multicomponent systems.

This modeling was done with the purpose of designing separation-purification process for valorization of the gaseous effluent of the torrefaction process for bio-sourced chemicals. Nevertheless, we are confident that this approach, developed here for a specific application, can be generic to describe other complex thermodynamic systems including reactive components.

\section{Acknowledgments}

The authors gratefully acknowledge financial support by the French Agence Nationale de la Recherche and also the partners of the INVERTO project (ANR-12-BIME-0008-04): CEA (Grenoble, France), CIRAD (Montpellier, France) and PCAS (Longjumeau, France).

\section{Nomenclature}

\section{Mathematical symbols}

$A_{i, j}, A_{j, i} \quad$ UNIQUAC binary interaction parameters of the components $\mathrm{i}$ and $\mathrm{j}(\mathrm{cal} / \mathrm{mol})$

$F_{o b j} \quad$ objective function

$K \quad$ chemical reaction equilibrium constant

$n \quad$ degree of polymerization

$n_{C} \quad$ number of components

$n_{\text {exp }} \quad$ number of experimental data point

$P \quad$ pressure of the system

$P_{i}^{S} \quad$ equilibrium vapor pressure of pure component i

$q_{i} \quad$ Van der Waals area parameter of the component $i$

$r_{i} \quad$ Van der Waals volume parameter of the component $\mathrm{i}$

$T \quad$ temperature of the system (K)

$T_{\text {ref }} \quad$ temperature at the reference state: $T_{r e f}=298.15 \mathrm{~K}$

$T_{\text {ref }} \quad T_{\text {ref }}=298.15^{\circ} \mathrm{C}$ $x_{i} \quad$ liquid molar fraction of the component $\mathrm{i}$

$y_{i} \quad$ vapor molar fraction of the component $\mathrm{i}$

$Z \quad$ lattice coordination number set equal to 10

\section{Components}

$\begin{array}{ll}A_{i} & \text { carboxylic acid } \\ A_{i 2} & \text { carboxylic acid dimer } \\ F A & \text { Formaldehyde } \\ F u & \text { Furfural } \\ F u A l & \text { Furfuryl Alcohol } \\ H F & \text { hemiformal } \\ H F_{n} & \text { poly(oxymethylene) hemiformal } \\ M E & \text { methanol } \\ M G & \text { methylene glycol } \\ M G_{n} & \text { poly(oxymethylene) glycol } \\ W & \text { water }\end{array}$

\section{Greek symbols}

$\gamma_{i} \quad$ activity coefficient of the component $i$

$\phi_{i} \quad$ UNIQUAC volume fraction of the component $\mathrm{i}$

$\phi_{i}^{V, S} \quad$ vapor fugacity coefficient of pure constituant i at

$\phi_{i}^{V} \quad$ vapor fugacity coefficient of constituant $i$ in the mixture

$\tau_{i, j}, \tau_{j, i}$ binary interaction characteristic energy parameters of the components $\mathrm{i}$ and $\mathrm{j}$

$\theta_{i} \quad$ UNIQUAC area fraction of the component $\mathrm{i}$

\section{Subscripts}

calc calculated

exp experimental

$i, j, k \quad$ index of the components

$l \quad$ index of the experimental points

ref reference state

\begin{tabular}{ll}
\multicolumn{2}{l}{ Superscripts } \\
bubble & bubble point \\
$C$ & combinatorial term \\
dew & dew point \\
$R$ & residual term \\
$S$ & saturation point
\end{tabular}

\section{References}

[1] M.J. Prins, K.J. Ptasinski, F.J. Janssen, Torrefaction of wood: part 2. analysis of products, J. Anal. Appl. Pyrolysis 77 (1) (2006) 35-40, http://dx.doi.org/ 10.1016/j.jaap.2006.01.001. URL, http://www.sciencedirect.com/science/ article/pii/S0165237006000167.

[2] T. Nocquet, C. Dupont, J.-M. Commandre, M. Grateau, S. Thiery, S. Salvador, Volatile species release during torrefaction of wood and its macromolecular constituents: part 1 experimental study, Energy 72 (2014) 180-187, http:/ dx.doi.org/10.1016/j.energy.2014.02.061. URL, http://www.sciencedirect.com/ science/article/pii/S0360544214001959.

[3] T. Nocquet, C. Dupont, J.-M. Commandre, M. Grateau, S. Thiery, S. Salvador Volatile species release during torrefaction of biomass and its macromolecular constituents: part 2 modeling study, Energy 72 (2014) 188-194, http:/ dx.doi.org/10.1016/j.energy.2014.05.023. URL, http://www.sciencedirect.com/ science/article/pii/S0360544214005726.

[4] K. Svoboda, M. Pohorely, M. Hartman, J. Martinec, Pretreatment and feeding of biomass for pressurized entrained flow gasification, Fuel Process. Technol. 90 (5) (2009) 629-635, http://dx.doi.org/10.1016/j.fuproc.2008.12.005. URL, http://www.sciencedirect.com/science/article/pii/S0378382008003287.

[5] K.J. Ptasinski, M.J. Prins, A. Pierik, Exergetic evaluation of biomass gasification, Energy 32 (4) (2007) 568-574, http://dx.doi.org/10.1016/j.energy.2006.06.024. URL, http://www.sciencedirect.com/science/article/pii/ S0360544206001836.

[6] J. Shankar Tumuluru, S. Sokhansanj, J.R. Hess, C.T. Wright, R.D. Boardman, Review: a review on biomass torrefaction process and product properties for energy applications, Ind. Biotechnol. 7 (2011) 384-401, http://dx.doi.org/ 10.1089/ind.2011.7.384. URL, http://online.liebertpub.com/doi/abs/10.1089/ ind.2011.7.384.

[7] M.J. Prins, K.J. Ptasinski, F.J. Janssen, More efficient biomass gasification via 
torrefaction, Energy 31 (15) (2006) 3458-3470, http://dx.doi.org/10.1016/ j.energy.2006.03.008. URL, http://www.sciencedirect.com/science/article/pii/ S036054420600065X.

[8] E.M. Fisher, C. Dupont, L.I. Darvell, J.M. Commandre, A. Saddawi, J.M. Jones, M. Grateau, T. Nocquet, S. Salvador, Combustion and gasification characteristics of chars from raw and torrefied biomass, Bioresour. Technol. 119 (2012) 157-165, http://dx.doi.org/10.1016/j.biortech.2012.05.109. URL, http://www. sciencedirect.com/science/article/pii/S0960852412008668.

[9] J. Ratte, E. Fardet, D. Mateos, J.-S. Hery, Mathematical modelling of a continuous biomass torrefaction reactor: torspyd column, Biomass Bioenergy 35 (8) (2011) 3481-3495, http://dx.doi.org/10.1016/j.biombioe.2011.04.045. URL, http://www.sciencedirect.com/science/article/pii/S0961953411002479.

[10] J.-M. Commandre, A. Leboeuf, Volatile yields and solid grindability after torrefaction of various biomass types, Environ. Prog. Sustain. Energy.

[11] M. Nala, E. Auger, I. Gedik, N. Ferrando, M. Dicko, P. Paricaud, F. Volle, J.P. Passarello, J.-C. de Hemptinne, P. Tobaly, P. Stringari, C. Coquelet, D. Ramjugernath, P. Naidoo, R. Lugo, Vapour-liquid equilibrium (VLE) for the systems furan $\hat{A}+\hat{A}$ n-hexane and furan $\hat{A}+\hat{A}$ toluene. measurements, data treatment and modeling using molecular models, Fluid Phase Equilibria 337 (0) (2013) 234-245, http://dx.doi.org/10.1016/j.fluid.2012.08.005. URL, http://www.sciencedirect.com/science/article/pii/S0378381212003585.

[12] T.M. Soria, A. Andreatta, S. Pereda, S.B. Bottini, Thermodynamic modeling of phase equilibria in biorefineries, Fluid Phase Equilibria 302 (1) (2011) 1-9.

[13] M. Detcheberry, P. Destrac, X.-M. Meyer, J.-S. Condoret, Phase equilibria of aqueous solutions of formaldehyde and methanol: improved approach using uniquac coupled to chemical equilibria, Fluid Phase Equilibria 392 (0) (2015) 84-94, http://dx.doi.org/10.1016/j.fluid.2015.02.011. URL, http://www. sciencedirect.com/science/article/pii/S0378381215000692.

[14] G. Maurer, Phase equilibria in chemical reactive fluid mixtures, Fluid Phase Equilibria 116 (1-2) (1996) 39-51, http://dx.doi.org/10.1016/0378-3812(95) 02870-6. URL, http://www.sciencedirect.com/science/article/pii/ 0378381295028706 .

[15] T. Nocquet, Torrefaction du bois et de ses constituants: experiences et modelisation des rendements en matieres volatiles, 2012. URL, http://www. theses.fr/2012INPT0159.

[16] W.-H. Chen, H.-C. Hsu, K.-M. Lu, W.-J. Lee, T.-C. Lin, Thermal pretreatment of wood (lauan) block by torrefaction and its influence on the properties of the biomass, Energy 36 (5) (2011) 3012-3021, http://dx.doi.org/10.1016/j.energy.2011.02.045. URL, http://www.sciencedirect.com/science/article/pii/ S0360544211001411.

[17] R.B. Bates, A.F. Ghoniem, Biomass torrefaction: modeling of volatile and solid product evolution kinetics, Bioresour. Technol. 124 (2012) 460-469, http:// dx.doi.org/10.1016/j.biortech.2012.07.018. URL, http://www.sciencedirect. com/science/article/pii/S0960852412010656.

[18] J. Vidal, Thermodynamique: Application au genie chimique et a l'industrie petroliere, Publications de l'Institut franÂß̧ais du petrole, Editions Technip, 1997.

[19] D.S. Abrams, J.M. Prausnitz, Statistical thermodynamics of liquid mixtures: a new expression for the excess gibbs energy of partly or completely miscible systems, AIChE J. 21 (1) (1975) 116-128, http://dx.doi.org/10.1002/ aic.690210115. URL, http://onlinelibrary.wiley.com/doi/10.1002/aic. 690210115/abstract.

[20] M. Albert, B. Coto Garcia, C. Kuhnert, R. Peschla, G. Maurer, Vapor liquid equilibrium of aqueous solutions of formaldehyde and methanol, AIChE J. 46 (2000) 1676-1687, http://dx.doi.org/10.1002/aic.690460818.

[21] A. Fredenslund, R.L. Jones, J.M. Prausnitz, Group-contribution estimation of activity coefficients in nonideal liquid mixtures, AIChE J. 21 (6) (1975) 1086-1099.

[22] R. Rowley, W. Wilding, J. Oscarson, Y. Yang, N. Zundel, T. Daubert, R. Danner, Dippr data compilation of pure chemical properties, design institute for physical properties, AIChE J., URL http://www/aiche.org/dippr.

[23] M. Abbott, H. Van Ness, Thermodynamics of solutions containing reactive species: a guide to fundamentals and applications, Fluid Phase Equilibria 77 (1992) 53-119.

[24] J. Gmehling, U. Onken, W. Arlt, P. Grenzheuser, U. Weidlich, B. Kolbe, J. Rarey, Vapor-liquid Equilibrium Data Collection Part 5: Carboxylic Acids, Anhydrides, Esters, Chemistry Data Series, Vol. 1, DECHEMA, 2001.

[25] S. Miyamoto, S. Nakamura, Y. Iwai, Y. Arai, Measurement of isothermal vaporliquid equilibria for monocarboxylic acid + monocarboxylic acid binary systems with a flow-type apparatus, J. Chem. Eng. Data 46 (2) (2001) 405-409, http://dx.doi.org/10.1021/je000205n.

[26] A. Tamir, C. Dragoescu, A. Apelblat, J. Wisniak, Heats of vaporization and vapor-liquid equilibria in associated solutions containing formic acid, acetic acid, propionic acid and carbon tetrachloride, Fluid Phase Equilibria 10 (1) (1983) 9-42, http://dx.doi.org/10.1016/0378-3812(83)80002-8. URL, http:// www.sciencedirect.com/science/article/pii/0378381283800028.

[27] Z. Univ, S. Shihuachang, J. Chem. Eng. (China) 2(87).

[28] L. Fele, V. Grilc, Separation of furfural from ternary mixtures, J. Chem. Eng. Data 48 (3) (2003) 564-570.

[29] A. Rius, J. Otero, A. Macarron, Equilibres liquide-vapeur de melanges binaires donnant une reaction chimique: systemes methanol-acide acetique; ethanolacide acetique; n-propanol-acide acetique; n-butanol-acide acetique, Chem. Eng. Sci. 10 (1) (1959) 105-111.

[30] S. Amer Amezaga, J. Fernandez Biarge, An. Quimica 69(569).

[31] I. Malijevska, M. Sysova, D. Vlckova, Collect. Czech. Chem. Commun. 51 (1986) 194.

[32] M. Kato, H. Yoshikawa, M. Yamaguchi, Vapor-liquid equilibrium measurements of three binary systems made of formic acid, acetic acid and propionic acid by the dew-bubble point temperature method, Fluid Phase Equilibria 54 (1990) 47-56, http://dx.doi.org/10.1016/0378-3812(90)85069-M. URL, http:// www.sciencedirect.com/science/article/pii/037838129085069M.

[33] O. Mikhailova, N. Markuzin, Equilibrium of liquid-associated vapor in formicacid acetic-acid and acetic-acid propionic-acid systems at 40-degrees-c, Zhurnal Obshchei Khimii 52 (10) (1982) 2164-2166.

[34] H. Brusset, L. Kaiser, J. Hocquel, Chim. Ind. Genie Chim., 99(207).

[35] R. Gilmont, D.F. Othmer, Composition of vapors from boiling binary solutions, Ind. Eng. Chem. 36 (11) (1944) 1061-1064.

[36] T. Ito, F. Yoshida, Vapor liquid equilibria of water lower fatty acid systems: water formic acid, water acetic acid and water propionic acid, J. Chem. Eng. Data 8 (1963) 315-320, http://dx.doi.org/10.1021/je60018a012.

[37] Y.A. Tsirlin, Sb. Tr. Vnii Gidroliza Rastit. N. Mater. 18 (1969) 190.

[38] M.G. Myles, R. Wingard, Calculating activity coefficients, Ind. Eng. Chem. 53 (3) (1961) 219-222

[39] K. Andreev, Y. A. Tsirlin, Zhurnal Obshchei Khimii 27(402).

[40] X. Ni, Z. Wang, J. Chem. Eng. (China) 3(50).

[41] M.S. Sunder, D. Prasad, Phase equilibria of water + furfural and dichloromethane+ n-hexane, J. Chem. Eng. Data 48 (2) (2003) 221-223.

[42] A. Roldyrev, V. Komarov, V. Krichevtsov, Zhurnal Obshchei Khimii 46(2338).

[43] V. Vasileva, S. Fedotova, Y. Tsirlin, Sb. Tr. Vnii Gidroliza Rastit. 93.

[44] A. Apelblat, F. Kohler, Excess gibbs energy of methanol+ propionic acid and of methanol+ butyric acid, J. Chem. Thermodyn. 8 (8) (1976) 749-756.

[45] K. Kojima, K. Tochigi, H. Seki, K. Watase, Kagaku Kogaku 32(149).

[46] V. Olevsky, I. Golubev, Tr. Giap. Vyp. 6(45).

[47] M. Albert, I. Hahnenstein, H. Hasse, G. Maurer, Vapor liquid equilibrium of formaldehyde mixtures: new data and model revision, AIChE J. 42 (1996) 1741-1752, http://dx.doi.org/10.1002/aic.690420625.

[48] M. Albert, B.C. Garcia, C. Kreiter, G. Maurer, Vapor liquid and chemical equilibria of formaldehyde water mixtures, AIChE J. 45 (1999) 2024-2033.

[49] I. Hahnenstein, M. Albert, H. Hasse, C.G. Kreiter, G. Maurer, Nmr spectroscopic and densimetric study of reaction kinetics of formaldehyde polymer formation in water, deuterium oxide, and methanol, Ind. Eng. Chem. Res. 34 (2) (1995) 440-450, http://dx.doi.org/10.1021/ie00041a003.

[50] J. Winkelman, O. Voorwinde, M. Ottens, A. Beenackers, L. Janssen, Kinetics and chemical equilibrium of the hydration of formaldehyde, Chem. Eng. Sci. 57 (19) (2002) 4067-4076.

[51] M. Albert, B.C. Garcia, C. Kreiter, G. Maurer, Vapor liquid and chemical equilibria of formaldehyde water mixtures, AIChE J. 45 (9) (1999) 2024-2033.

[52] H. Sawistowski, P.A. Pilavakis, Vapor-liquid equilibrium with association in both phases. multicomponent systems containing acetic acid, J. Chem. Eng. Data 27 (1) (1982) 64-71.

[53] V. Aristovich, A. Levin, A. Morachevsky, Tr. Vses. Nauch. Issled. Inst. Neft. Protsessov 84(5).

[54] T. Kushner, G. Tatsievskaya, V. Irzun, Russ. J. Phys. Chem. A 40(3010).

[55] J. Conti, D. Othmer, R. Gilmont, Composition of vapors from boiling binary solutions. systems containing formic acid, acetic acid, water, and chloroform, J. Chem. Eng. Data 5 (3) (1960) 301-307.

[56] K. Andreev, Y. Tsirlin, Zh. Prikl. Khim. 27(402). 